\title{
The Formation of Stellar Galactic Nuclei through Dissipative Gas Dynamics
}

\author{
K. Bekki \\ A School of Physics, University of New South Wales, Sydney 2052, Australia. \\ Email: bekki@phys.unsw.edu.au
}

Received 2007 February 23, accepted 2007 June 22

\begin{abstract}
It is a long-standing and remarkable problem as to how stellar galactic nuclei (SGN) were formed in the central region of galaxies. In order to elucidate the formation processes of SGN, we numerically investigate gas dynamics, star formation, and chemical evolution in the central 1-1000 pc of gas disks embedded by galactic stellar spheroids. The main results of the present numerical study are: (a) SGN can be formed from dissipative, repeated merging of massive stellar and gaseous clumps that have typical masses of $10^{5}-10^{6} \mathrm{M}_{\odot}$ and are developed from nuclear gaseous spiral arms owing to local gravitational instability. Typically $\sim 5 \%$ of the masses of their host spheroids can be transfered to the central $\sim 50 \mathrm{pc}$ and thus become SGN. (b) SGN have very flattened shapes, and rotational kinematics and central velocity dispersions much smaller than those of their host spheroids. These structural and kinematic characteristics do not depend on model parameters such as masses of spheroids $\left(M_{\mathrm{sph}}\right)$ and initial gas mass fraction $\left(f_{\mathrm{g}}\right)$. (c) Stellar populations of SGN can show a wide rage of ages and metallicities, because SGN are formed from massive clumps with different star-formation and chemical-evolution histories. The mean metallicities of SGN can be significantly higher than those of their host spheroids. (d) More massive, higher density SGN can be formed in spheroids with higher surface brightness. Furthermore there can be a threshold value $(\sim 0.2)$ of $f_{\mathrm{g}}$ below which massive SGN are less likely to be formed in the central gas disks of spheroids. (e) More massive spheroids can have more massive, more metal-rich and higher-density SGN, because star formation and chemical enrichment proceed more efficiently owing to the less dramatic suppression of star formation by supernovae feedback effects in more massive spheroids.

Based on these results, we discuss correlations between the physical properties of SGN and those of their host galaxies, structural and kinematic properties of SGN of dwarf elliptical galaxies and the origin of very massive star clusters such as $\omega$ Cen and ultra-compact dwarf galaxies.
\end{abstract}

Keywords: galaxies: nuclei — galaxies: irregular — galaxies: starburst — galaxies: star clusters — galaxies: structure

\section{Introduction}

Recent Hubble Space Telescope (HST) photometric observations have revealed that a significant fraction of late-type galaxies have very compact stellar systems - which are referred to as stellar galactic nuclei (SGN) in this paper in the very center of these galaxies (Phillips et al. 1996; Carollo, Stiavelli \& Mack 1998; Matthews et al. 1999; Böker et al. 2002, 2004; Walcher et al. 2005; Côte et al. 2006; Seth et al. 2006). I-band absolute magnitudes of these SGN are observed to range from -8 to -14 mag and thus some of them are much more luminous than those of globular clusters (GCs) in the Galaxy and M31 (Böker et al. 2004). Physical properties of SGN have been suggested to correlate with those (e.g. masses and surface brightness) of the host galaxies (Böker et al. 2004). Similarity and differences between SGN and GCs have raised a number of key questions as to how such compact and massive stellar systems are formed in the central regions of galaxies (e.g. Böker et al. 2004; Bekki et al. 2004; Milosavljević 2004).
Spectrophotometric studies of nucleated dwarf galaxies (dE,Ns) in nearby clusters of galaxies have also provided new clues to the origin of SGN in these dE,Ns (e.g. Stiavelli et al. 2001; Geha, Guhathakurta \& van der Marel 2002; Lotz, Miller \& Ferguson 2004). Stiavelli et al. (2001) found that structural properties of SGN in dE,Ns are very similar to those of exponential bulges of spiral galaxies. Geha et al. (2002) investigated radial profiles of structural and kinematics properties of nuclear regions of six dE,Ns in the Virgo Cluster and found that massive black holes with the masses of more than $10^{7} \mathrm{M}_{\odot}$ are highly unlikely to be within the nuclear regions of these dE,Ns. Lotz et al. (2004) found that the stellar envelopes of dE,Ns are $0.1-0.2$ mag redder in $V-I$ than their SGN and thus suggested that star-formation histories can be quite different between the envelopes and SGN. These recent observations, combined with previous ones on dE,Ns (e.g. Binggeli \& Cameron 1991; Ferguson \& Binggeli 1994), have given some constraints on any theory of SGN formation in $\mathrm{dE}, \mathrm{Ns}$. 
Since 'ultra-compact dwarf' (UCD) galaxies were discovered in an 'all-object' spectroscopic survey centred on the Fornax Cluster (Drinkwater et al. 2000; Philips et al. 2001), physical relations between UCDs and SGN have been extensively discussed (e.g. Drinkwater et al. 2003; Mieske, Hilker \& Infante 2004; De Propris et al. 2005; Drinkwater et al. 2005; Jones et al. 2006; Gregg et al. 2007). Drinkwater et al. (2003) revealed that the locations of UCDs on the $M_{\mathrm{V}}-\sigma_{0}$ relation, where $M_{\mathrm{V}}$ and $\sigma_{0}$ are $V$-band magnitude and central velocity dispersion, respectively, are quite different from those of GCs and dEs. They also found that $\mathrm{dE}$,Ns can be in similar locations on the $M_{\mathrm{V}}-\sigma_{0}$ relation as UCDs, if their stellar envelope are totally stripped with their SGN being left as they are. De Propris et al. (2005) have revealed that UCDs are more extended and have higher surface brightness than SGN of typical dE,Ns and accordingly suggested that evolutionary links between SGN and UCDs are less likely.

Although these recent observations have provided vital clues to the better understanding of the formation of SGN, only a few theoretical works have thus far investigated the formation processes of SGN (e.g. Tremaine, Ostriker \& Spitzer 1975; Oh \& Lin 2000; Fellhauer \& Kroupa 2002; Bekki et al. 2004). One of the most extensively discussed scenarios is that these SGN are formed from the merging of smaller stellar clusters like GCs in the central region of galaxies (e.g. Oh \& Lin 2000; Bekki et al. 2004). These numerical works, which are based on dissipationless models of merging star clusters, provide some predictions of the structural and kinematic properties of SGN. These purely dissipationless models did not discuss at all how gaseous dissipation and star formation are important in the formation of SGN, though a number of spectrophotometric observations have already provided possible evidence for very young stellar populations in SGN in some nucleated galaxies (Caldwell \& Bothun 1987; Bothun \& Mould 1988; Böker et al. 2004; Lotz et al. 2004). It is thus still unclear whether gaseous dissipation and star formation play a vital role in SGN formation in the central regions of galaxies.

The purpose of this paper is to try to understand what physical roles gaseous dissipation and star formation can play in the formation of SGN. By using fully self-consistent chemodynamical simulations, we investigate how SGN are formed in the central $1-1000 \mathrm{pc}$ of gas disks embedded by stellar spheroids. We particularly investigate the dependences of structural, kinematic, and chemical properties of SGN on model parameters and thereby provide theoretical predictions that can be tested against recent observations, such as correlations between physical properties of SGN and those of their host galaxies (e.g. Böker et al. 2004), kinematic properties of dE,Ns (e.g. Geha et al. 2003), and scaling relations of SGN (e.g. Walcher et al. 2005). The present study is the very first step toward the better understanding of dissipative SGN formation and can be regarded as complementary to the previous ones based on dissipationless models (e.g. Oh \& Lin 2000; Bekki et al. 2004). The present study and our previous one (Bekki, Couch \& Shioya 2006) might well provide a vital clue to the origin of the observed correlations between physical properties of SGN and those of their host galaxies (e.g. Graham \& Guzmán 2003).

The plan of the paper is as follows: in Section 2 we describe our numerical models for SGN formation in gas disks of galactic spheroids; in Section 3 we present the numerical results on SGN formation and their dependences on model parameters; in Section 4 we provide several theoretical predictions obtained in the present study and thereby discuss the origin of SGN in galaxies with different Hubble types; we summarize our conclusions in Section 5 .

\section{The Model}

We investigate the dynamical evolution of stellar and gaseous components in the central 1-1000 pc of a galaxy, via chemodynamical simulations carried out on a GRAPE board (Sugimoto et al. 1990). Since the numerical methods and techniques we use for the GRAPE system have already been described in detail elsewhere (Bekki \& Shioya 1998, 1999), we give only a brief review here.

\subsection{Stellar Spheroids}

Our primary focus is on the SGN formation in small galactic bulges in late-type, gas-rich galaxies and in bright dwarf galaxies. A significant fraction of these spheroidal components are observed to have exponential luminosity profiles (e.g. Ichikawa, Wakamatsu \& Okamura 1986; Andredakis \& Sanders 1994). Therefore the projected radial density profile $\left(\Sigma_{\mathrm{sph}}\right)$ of the stellar spheroid with the mass of $M_{\text {sph }}$ and the scale length of $a_{\text {sph }}$ is assumed to be an exponential one:

$$
\Sigma_{\mathrm{sph}}(R)=\Sigma_{\mathrm{sph}, 0} \times \exp \left(-R / a_{\mathrm{sph}}\right),
$$

where $\Sigma_{\mathrm{sph}, 0}$ and $R$ are the central surface density and the projected radius from the center of the spheroid, respectively. In order to derive the three dimensional (3D) density field $-\rho_{\mathrm{sph}}(r)$ where $r$ is the distance from the center of the spheroid) from $\Sigma_{\mathrm{sph}}$ - we can use the following formula (Binney \& Tremaine 1987):

$$
\rho_{\mathrm{sph}}(r)=-\frac{1}{\pi} \int_{r}^{\infty} \frac{\mathrm{d} \Sigma_{\mathrm{sph}}(R)}{\mathrm{d} R} \frac{\mathrm{d} R}{\left(R^{2}-r^{2}\right)^{1 / 2}} .
$$

We numerically estimate the $\rho_{\text {sph }}(r)$ profile for a given $\Sigma_{\mathrm{sph}}(R)$ in a model with $M_{\mathrm{sph}}$ and $a_{\mathrm{sph}}$. Guided by recent observations on the scaling relation between $\Sigma_{\text {sph,0 }}$ and $M_{\text {sph }}$ (i.e. $\Sigma_{\text {sph }, 0} \propto M_{\text {sph }}^{0.54}$, Kauffmann et al. 2003), we adopt the following $M_{\mathrm{sph}}-a_{\mathrm{sph}}$ relation

$$
a_{\mathrm{sph}}=C_{0} \times M_{\mathrm{sph}}^{0.23},
$$

where $C_{0}$ is chosen such that the mass and the scale length of the Galactic exponential bulge can be reproduced reasonably well. For example, this equation gives $a_{\mathrm{sph}}=200 \mathrm{pc}$ for $M_{\mathrm{sph}}=10^{9} \mathrm{M}_{\odot}$. The models with $2.5 \times 10^{7} \mathrm{M}_{\odot} \leq M_{\mathrm{sph}} \leq 5 \times 10^{9} \mathrm{M}_{\odot}$ are investigated in the present study. 
The way of constructing the dynamically equilibrium models of spheroidal components with gas disks in the present study is essentially similar to previous works (e.g. Noguchi 1991). The spheroid is assumed to be supported purely by velocity dispersion and its dispersion is assumed to be isotropic. We therefore estimate the velocities of old stellar particles from the gravitational potential at the positions where they are located. In detail, we first calculate the one-dimensional isotropic dispersion according to the (local) virial theorem:

$$
\sigma^{2}(r)=-\frac{U(r)}{3},
$$

where $U(r)$ is the gravitational potential at the position $r$. Then we allocate a velocity to stellar particle so that the distribution of velocities of these particles can have a Gaussian form with a dispersion equal to $\sigma^{2}(r)$. After giving $\sigma^{2}(r)$ for each stellar particle, only the spheroidal component is dynamically evolved with the gas disk fixed for 10 dynamical time scales. The spheroidal component can reach a new equilibrium state during 10 dynamical time scales. This relaxed spheroidal component is used as an initial model. Since this paper focuses on galactic nuclear regions (1-1000 pc), where the luminous components of galaxies gravitationally dominate the systems, the model does not include a dark matter component.

\subsection{Gas Disks}

The central regions of late-type, gas-rich galaxies are more likely to be dominated by molecular gas $\left(\mathrm{H}_{2}\right)$ rather than Hi (e.g. Tacconi \& Young 1986). Therefore the gas disk is modeled as a collection of discrete gas clouds (corresponding to giant molecular clouds, GMCs) that follow the observed mass-size relationship of GMCs (Larson 1981):

$$
m_{\mathrm{cl}}=C_{\mathrm{cl}} \times r_{\mathrm{cl}}^{2},
$$

where $m_{\mathrm{cl}}$ and $r_{\mathrm{cl}}$ are the mass and the size of a GMC, respectively. For the adopted constant $C_{\mathrm{cl}}$, a GMC with $m_{\mathrm{cl}}=10^{4} \mathrm{M}_{\odot}$ has $r_{\mathrm{cl}}=3 \mathrm{pc}$. The thin gas disk composed of these clouds is assumed to have an exponential profile with the scale length being the same as $a_{\text {sph. }}$. The mass ratio of the gas disk to the spheroid is regarded as a free parameter $\left(f_{\mathrm{g}}\right)$ that ranges from 0.02 to 0.5 . We thus can investigate formation processes of SGN both for gas-poor galaxies and for gas-rich ones.

Every pair of two overlapping gas clouds is made to collide with the same restitution coefficient (Hausman \& Roberts 1984) and lose its random kinematic energy through gaseous dissipation. Although the adopted method of 'sticky particles' has been proven to be capable of addressing successfully some aspects of hydrodynamical interactions in the interstellar medium (ISM) for disk galaxies (e.g. Hausman \& Roberts 1984; Combes \& Gerin 1985), it has some disadvantages in dealing with the more realistic physical processes of the ISM, such as the hydrodynamical interaction between hot interstellar gas (with the temperature of $10^{6} \mathrm{~K}$ ) and GMCs (e.g. evaporation of GMCs by the hot gas). Each gaseous particle is assumed to have a rotational velocity determined by the mass profile of stars and gas. The initial gas disk is assumed to be dynamically cold and thus to have no vertical nor radial velocity dispersion.

\subsection{Star Formation and Chemical Evolution}

\subsubsection{Feedback from Supernovae}

Field star formation is modeled by converting the collisional gas particles into collisionless new stellar particles according to the star-formation algorithm described below. We adopt the Schmidt law (Schmidt 1959) with exponent $\gamma=1.5(1.0<\gamma<2.0$, Kennicutt 1998) as the controlling parameter of the rate of star formation. The amount of gas consumed by star formation for each gas particle in each time step is given as:

$$
\dot{\rho}_{\mathrm{g}} \propto \rho_{\mathrm{g}}^{\gamma}
$$

where $\rho_{\mathrm{g}}$ is the gas density around each gas particle. We convert a gas particle into a new field star only if the local mean gas density $\rho$ exceeds the threshold gas density of $\sim 10^{3} \mathrm{M}_{\odot} \mathrm{pc}^{-3}$. This threshold for mean cloud density corresponds to the cloud's core density of $\sim 10^{5} \mathrm{M}_{\odot} \mathrm{pc}^{-3}$ for reasonable cloud structures and therefore is consistent with theoretical predictions by Elmegreen (2002). These stars formed from gas are called 'new stars' (or 'young stars') whereas stars initially within a spheroid are called 'old stars' throughout this paper.

The dynamical evolution of the interstellar medium (ISM) can be significantly influenced by supernova explosions; the effects of supernova explosions on the ISM depend on the initial mass function (IMF) adopted in the present study. For the stellar population formed in a GMC, we assume an IMF described as $\psi\left(m_{i}\right)=A m_{i}^{-s}$, where $m_{i}$ is the initial mass of each individual star and the slope of $s=2.35$ corresponds to the Salpeter IMF. The normalization factor $A$ is a function of $m_{\mathrm{cl}}, m_{1}$ (lower mass cut-off) and $m_{\mathrm{u}}$ (upper one):

$$
A=\frac{m_{\mathrm{cl}} \times(2-s)}{m_{\mathrm{u}}^{2-s}-m_{1}^{2-s}} .
$$

$m_{\mathrm{u}}$ is a parameter and set to be $100 \mathrm{M}_{\odot}$ for most models whereas $m_{\mathrm{L}}$ is regarded as a key free parameter in the present study. We shows the results of the canonical models with $m_{1}=0.1 \mathrm{M}_{\odot}$ and $m_{\mathrm{u}}=100 \mathrm{M}_{\odot}$ and the topheavy IMF ones with $m_{1}=1.0 \mathrm{M}_{\odot}$ and $m_{\mathrm{u}}=100 \mathrm{M}_{\odot}$. The mass fraction of stars that can explode as Type-II supernovae (i.e. $m_{\mathrm{I}}>8 \mathrm{M}_{\odot}$ ) is $14 \%$ for the canonical model and $35 \%$ for the top-heavy IMF ones. About $10 \%$ of the total energy of one supernova $\left(\sim 10^{51} \mathrm{erg}\right)$ is assumed to be converted into the kinematic energy of gas clouds around the supernova (Thornton et al. 1998).

Since we investigate the dynamical evolution of galactic nuclear regions for less than $\sim 0.7$ Gyr for most models, only feedback effects from Type II supernovae (SNe II) can be considered in the present study. Feedback effects from Type-Ia supernovae and stellar winds from low mass stars cannot be important within less than 1 yr (Bekki \& Shioya 1999). Some fraction of the supernovae energy 
$\left(E_{\mathrm{SN}, i}\right)$ of the $i$ th stellar particle is assumed to be returned to gas particles (ISM) and then to change the kinetic energy of gas particles around the $i$ th stellar particle. Each $j$ th gas particle with the mass of $m_{g, j}$ around an $i$ th stellar particle can receive a velocity perturbation $\left(\Delta v_{\mathrm{SN}, j}\right)$ directed radially away from the $i$ th stellar particle. $\Delta v_{\mathrm{SN}, j}$ satisfies the relation:

$$
f_{\mathrm{SN}} E_{\mathrm{SN}, i}=\frac{1}{2} \sum_{j=1}^{N_{\mathrm{ne}, i}} m_{g, j}\left(\Delta v_{\mathrm{SN}, j}\right)^{2},
$$

where $f_{\mathrm{SN}}$ represents the fraction of supernovae energy returned to ISM, $N_{\text {nei, } i}$ is the total number of gas cloud particles that are located within the cloud radius around an $i$ th stellar particle. $f_{\mathrm{SN}}$ is fixed at a reasonable value of 0.1 which is derived by numerical studies on the influence of supernovae explosion on ISM by Thornton et al. (1998). $E_{\mathrm{SN}, i}$ is $10^{51} \mathrm{erg}$ for all models.

\subsubsection{Chemical Enrichment}

Chemical enrichment through star formation and supernova feedback during the SGN formation and evolution is assumed to proceed locally in the present study. We assign the metallicity of original gas particle to the new stellar particle and increase the metals of each neighbor gas particle with the total number of neighbor gas particles equal to $N_{\text {gas }}$, according to the following equation for chemical enrichment:

$$
\Delta M_{\mathrm{Z}}=\frac{Z_{\mathrm{i}} R_{\mathrm{met}} m_{\mathrm{s}}+\left(1.0-R_{\mathrm{met}}\right)\left(1.0-Z_{\mathrm{i}}\right) m_{\mathrm{s}} y_{\mathrm{met}}}{N_{\mathrm{gas}}}
$$

where the $\Delta M_{\mathrm{Z}}$ represents the increase of metals for each gas particle. $Z_{\mathrm{i}}, R_{\mathrm{met}}, m_{\mathrm{s}}$, and $y_{\mathrm{met}}$ in the above equation represent the metallicity of the new stellar particle (or that of original gas particle), the fraction of gas returned to interstellar medium, the mass of the new star, and the chemical yield, respectively. The first term in this equation is from metals that stars initially have (i.e. before nucleosynthesis) whereas the second is from newly synthesized ones within the stars.

The final metallicity of a galaxy after gas consumption by star formation becomes similar to the adopted chemical yield $\left(y_{\text {met }}\right)$ for the present chemical evolution model, with an approximation of instantaneous chemical mixing. Since we consider the evolution of low-mass galaxies, the chemical yield $\left(y_{\text {met }}\right)$ should be as low as the observed stellar metallicities of low-mass galaxies (e.g. Côte et al. 2000). The values of $R_{\text {met }}$ and $y_{\text {met }}$ are thus set to be 0.3 and 0.005 , respectively. Initial gaseous metallicity $\left(Z_{i}\right.$ or $\left.[\mathrm{Fe} / \mathrm{H}]_{\mathrm{i}}\right)$ is assumed to be the same as the initial stellar one in each model and it is chosen such that it can be consistent with the observed mass-metallicity relation (e.g. Mould 1984). Guided by observational and theoretical results on the mass-metallicity (or luminosity-metallicity) relations for elliptical and dwarf galaxies (e.g. Dekel \& Silk 1986; Davies et al. 1987; Mateo 1998), we adopt the following relation:

$$
Z_{\mathrm{i}}=C_{\mathrm{m}} M_{\mathrm{sph}}^{0.25}
$$

where $C_{\mathrm{m}}$ is chosen such that $Z_{\mathrm{i}}=0.02$ (i.e. solar metallicity) for $M_{\mathrm{sph}}=6.0 \times 10^{12} \mathrm{M}_{\odot}$ (i.e. the Galactic luminous mass).

\subsection{Influences of Central MBHs}

Although our main purpose of this paper is not the formation of massive black holes (MBHs) in galactic nuclei, we investigate how MBHs dynamically influence the formation processes of SGN if there are MBHs in low-mass galaxies. A $\mathrm{MBH}$ with the mass of $M_{\mathrm{mbh}}$ in a galaxy is represented by a point-mass particle and accordingly the gravitational force between a particle and the $\mathrm{MBH}$ is proportional to $-M_{\mathrm{mbh}} / R_{\mathrm{mbh}}^{2}$, where $R_{\mathrm{mbh}}$ is the distance between the particle and the MBH. The present study cannot investigate tidal destruction of stars and clouds by MBHs, because the resolution of the simulations is not high enough to resolve sub-parsec scale dynamics of this tidal destruction. Although structural and kinematic changes of SGN by dynamical relaxation of stars interacting with MBHs can be important for SGN evolution, the present models do not allow us to investigate this owing to the adopted resolution of the simulations. Although our previous simulations investigated AGN feedback effects on gas dynamics in galaxies (e.g. Bekki, Shioya \& Whiting 2006), we do not intend to investigate these in the present paper.

\subsection{Gravitational Softening Length}

The main purpose of this paper is to investigate formation processes of $S G N$ for the central 1-1000 pc of galaxies rather than dynamical evolution of SGN via two-body relation effects after their formation. Therefore, the initial value of the gravitational softening length $\left(\epsilon_{\mathrm{g}}\right)$ of a selfgravitating system with the particle number of $N$ is chosen so that $\epsilon_{\mathrm{g}}$ is roughly the same as the mean particle separation at the half-mass radius $\left(R_{\mathrm{h}}\right)$ of the entire system (i.e. $\epsilon_{\mathrm{g}} \propto R_{\mathrm{h}} / N^{1 / 3}$ ). We confirm that if we use the formula shown in equation (8.1) of Binney \& Tremaine (1987) for the relaxation time scale $\left(t_{\text {relax }}\right)$, the simulated compact stellar systems within the central $100 \mathrm{pc}$ of galaxies have $t_{\text {relax }} \sim 2.7 \times 10^{8} \mathrm{yr}$. This order-of-magnitude estimation suggests that if we try to investigate long-term (>1Gyr) dynamical evolution of SGN via relaxation effects after their formation, different numerical models and codes (that can be run on GRAPE 6 systems rather than GRAPE 5 ones where GRAPE represents Gravity of PiPE) are necessary.

All the calculations related to the above chemodynamical evolution have been carried out on the GRAPE board (Sugimoto et al. 1990) of the GRAPE 5 systems at the Astronomical Data Analysis Center (ADAC) in the National Astronomical Observatory of Japan. The total number of particle is $10^{5}$ for old stars $\left(N_{\text {old }}\right)$ and $2 \times 10^{4}$ for initial gas $\left(N_{\text {gas }}\right)$ and the gravitational softening parameter is $20 \mathrm{pc}(9 \mathrm{pc})$ for $M_{\mathrm{sph}}=10^{9} \mathrm{M}_{\odot}\left(2.5 \times 10^{7} \mathrm{M}_{\odot}\right)$ in a simulation. The time integration of the equation of motion is performed by using the second-order leap-frog method and the total CPU time required for a simulation is 
about 42 hours for 3.1 Gyr evolution. In order to check the dependences of SGN formation on total particle number of simulations, we also run several higher-resolution models with $N_{\text {old }}=2 \times 10^{5}$ and $N_{\text {gas }}=2 \times 10^{4}$. We find that there are no significant differences in the results between the lower and higher resolution models. Structural and kinematic changes of SGN (e.g. evolution from more flattened systems into more spherical ones) via two-body relaxation will be investigated in our future study based on GRAPE 6 simulations.

\subsection{Main Points of Analysis}

We mainly investigate projected radial density profiles $\left(\mu_{\mathrm{s}}\right)$ of new and old stars in the central regions of spheroids. In order to compare the simulated profiles $\left(\mu_{\mathrm{S}}\right)$ with the observed $B$-band surface-brightness profiles $\left(\mu_{\mathrm{B}}\right)$, we use the following formula (Binney \& Merrifield 1998):

$$
\mu_{\mathrm{B}}=27.05-2.5 \log \left(I_{\mathrm{B}}\right),
$$

where $I_{\mathrm{B}}$ is the $B$-band surface brightness of a stellar system measured in units of $\mathrm{L}_{\odot} \mathrm{pc}^{-2}$. We estimate $I_{\mathrm{B}}$ from $\mu_{\mathrm{S}}$ for a given mass-to-light ratio $(M / L)$ in the $B$-band. $M / L$ can be significantly different between stellar populations with different ages for a given metallicity (e.g. Vazdekis et al. 1996). We therefore adopt two different $M / L$ for old and new stars. For old stars, we adopt the $M / L$ of a single stellar population (SSP) with $10 \mathrm{Gyr}$ and solar metallicity in the $B$-band:

$$
\left(M / L_{\mathrm{B}}\right)_{\mathrm{old}}=10 .
$$

For new stars, we adopt the following two values:

$$
\left(M / L_{\mathrm{B}}\right)_{\text {new }}=10 \text { or } 0.5 \text {, }
$$

where the former and the latter values correspond to a SSP of $10 \mathrm{Gyr}$ and solar metallicity and a SSP of $0.5 \mathrm{Gyr}$ and solar metallicity, respectively (Vazdekis et al. 1996). By comparing the models with $\left(M / L_{\mathrm{b}}\right)_{\text {new }}=10$ and $\left(M / L_{\mathrm{B}}\right)_{\text {new }}=0.5$, we can discuss how the aging of young stellar populations can control the $\mu_{\mathrm{B}}$ profiles.

Although spectroscopic observations have revealed kinematic properties of SGN, they have not yet derived typical ages and metallicities of SGN in galaxies (Walcher et al. 2005). Therefore, the above combination of SSPs for old and new stars should be considered as one of many possible candidates. We adopt the above set of SSPs because we would like to illustrate how surface brightness profiles of SGN depend on the ages of young stellar populations of SGN (e.g. Figure 6). It should be stressed here that the adopted combination of SSPs is not typical one. $M / L$ in the $B$-band can be smaller in stellar populations with younger ages for a given metallicity whereas it can be smaller in stellar populations with smaller metallicities for a given age (e.g. $M / L=0.30$ for SSP with $[\mathrm{Fe} / \mathrm{H}]=-1.68$ at $0.5 \mathrm{Gyr}$ whereas $M / L=0.53$ for SSP with $[\mathrm{Fe} / \mathrm{H}]=0.0$ in the SSP library by Vazdekis et al. 1996). This means that $B$-band surface brightness can be higher for SGN with younger and more metal-poor stellar populations. The uncertainty in $\mu_{\mathrm{B}}$ for each radial bin is estimated as $\mu_{\mathrm{B}} /\left[2\left(N_{i}-1\right)\right]^{1 / 2}$, where $N_{i}$ is the total number of particles in each bin. Since $N_{i}$ is typically larger than $\sim 100$ (depending on model and radius), the error in the simulated $\mu_{\mathrm{B}}$ can be small.

Two important parameters in SGN formation in the present study are $M_{\mathrm{sph}}$ and $f_{\mathrm{g}}$. We show the results of 21 representative models with different $M_{\text {sph }}$ and $f_{\mathrm{g}}$. The model parameters are summarized in Table 1. First, we show the results of the 'fiducial model' M1, because this model shows typical behaviours of SGN formation. Then we describe the parameter dependences of SGN formation processes and the physical properties of SGN. We also investigate the models with central MBHs with masses $\left(M_{\mathrm{mbh}}\right)$ equal to $0.006 M_{\mathrm{sph}}(\mathrm{M} 8)$ and $0.018 M_{\mathrm{sph}}$ (M9), though it is observationally less clear whether small bulges with exponential profiles and bright dwarf galaxies contain MBHs (e.g. Geha et al. 2002 for dE,Ns). The adopted values of $M_{\mathrm{mbh}}$ are consistent with observations by Magorrian et al. (1998).

The canonical IMF is adopted for all models except M10, 18, and 19. For the M18 model the total number of Type-II SNe per new stellar particle is smaller by a factor of 3.4, owing to the adopted smaller $m_{\mathrm{u}}$ $\left(=10 \mathrm{M}_{\odot}\right)$. Feedback effects of Type-II SNe in this model are significantly weaker than in the fiducial model. Stellar populations are dominated by low-mass stars in the M19 model with a 'bottom-heavy' IMF, where $m_{\mathrm{u}}=3 \mathrm{M}_{\odot}$ and $m_{1}=0.07 \mathrm{M}_{\odot}$. This lower-mass cut-off corresponds to the possible lowest mass of stars that can fuse $\mathrm{H}$ into $\mathrm{He}$ in their cores. We investigate models with different initialization of gas particle distributions (for fixed exponential profiles) by adopting different random number for random number generators. The results of model M21 are compared with those of the fiducial model M1 and thereby discussed in terms of the robustness of the present results.

We also investigate models in which stellar spheroids (i.e. M3 and M4) have lower or higher central surface brightness/density $\left(\Sigma_{\mathrm{sph}, 0}\right)$ compared with the fiducial model. We shows the results of: (a) the low surface brightness model (LSB) in which $\Sigma_{\mathrm{sph}, 0}$ is two magnitude lower than that of the fiducial one; (b) the high surface brightness one (HSB) in which $\Sigma_{\mathrm{sph}, 0}$ is one magnitude higher than that of the fiducial one. Results of the LSB model can be interpreted as the transformation from nonnucleated dwarf elliptical galaxies (dEs) into nucleated ones (dE,Ns). In the following, the time $T$ represents the time that has elapsed since the simulation starts and 'SGN' can also mean 'stellar galactic nucleus'.

\section{Results}

\subsection{Fiducial Model}

\subsubsection{Formation Processes}

Figures 1 and 2 show how a SGN is formed in the starforming gas disk embedded by the old stellar spheroid for the fiducial model M1. Numerous spiral arms composed only of gas clouds first develop owing to the 
Table 1. Model parameters

\begin{tabular}{llll}
\hline Model No. & $M_{\text {sph }}\left(\times 10^{9} \mathrm{M}_{\odot}\right)^{\mathrm{A}}$ & $f_{\mathrm{g}}{ }^{\mathrm{B}}$ & Comments \\
\hline M1 & 1.0 & 0.2 & fiducial model \\
M2 & 1.0 & 0.2 & no supernova feedback \\
M3 & 1.0 & 0.2 & LSB \\
M4 & 1.0 & 0.2 & HSB \\
M5 & 1.0 & 0.02 & \\
M6 & 1.0 & 0.1 & \\
M7 & 1.0 & 0.5 & \\
M8 & 1.0 & 0.2 & $M_{\text {mbh }}=0.006 M_{\text {sph }}$ \\
M9 & 1.0 & 0.2 & $M_{\text {mbh }}=0.018 M_{\text {sph }}$ \\
M10 & 1.0 & 0.2 & top-heavy IMF \\
M11 & 0.025 & 0.2 & \\
M12 & 0.1 & 0.2 & \\
M13 & 0.5 & 0.2 & \\
M14 & 0.1 & 0.02 & \\
M15 & 0.1 & 0.1 & \\
M16 & 0.1 & 0.5 & \\
M17 & 5.0 & 0.2 & more massive spheroid \\
M18 & 1.0 & 0.2 & $m_{\mathrm{u}}=10$ M $\odot$ \\
M19 & 1.0 & 0.2 & bottom-heavy IMF \\
M20 & 1.0 & 0.2 & smaller softening length \\
M21 & 1.0 & 0.2 & a different disk initialization \\
& & &
\end{tabular}

${ }^{A}$ Initial mass of a galactic spheroid.

B Initial gas mass fraction.

${ }^{\mathrm{C}} m_{\mathrm{l}}=0.07 \mathrm{M}_{\odot}$ and $m_{\mathrm{u}}=3 \mathrm{M}_{\odot}$.

stronger self-gravity of gas $\left(f_{\mathrm{g}}=0.2\right)$ as the gas disk rotates $(T=62 \mathrm{Myr})$. Because of the enhanced cloudcloud collisions rate and the resultant efficient gaseous dissipation within the gaseous spiral arms, small gas clumps composed of several tens of gas clouds can be formed within each spiral arm $(T=187 \mathrm{Myr})$. Some of these small gaseous clumps are massive, compact and strongly self-gravitating so that gas can be continuously converted into stars within the clumps $(T=187 \mathrm{Myr})$. These clumps can spiral in owing to dynamical friction against the stellar background of old stars (i.e. the stellar spheroid) and consequently merge with one another to form two very massive clumps composed mostly of new stars $(T=250 \mathrm{Myr})$. Numerous clumps located in the outer part of the gas disk are less massive so that dynamical friction alone cannot transfer them into the central $\sim 100$ pc. These outer clumps with new stars might well be identified either as compact star-forming regions or as super star clusters.

The two massive clumps developed within the central $200 \mathrm{pc}$ can interact with each other to form tidal arms composed only of new stars $(T=374 \mathrm{Myr})$. Their mutual tidal interaction result in the loss of orbital angular momentum of the clumps and therefore finally merge with each other to form a single compact stellar system in the central region of the stellar spheroid. ( $T=437 \mathrm{Myr})$. Although the single nucleus is initially not in the very center of the spheroid ( $T=437 \mathrm{Myr})$, dynamical friction between the nucleus and the stellar background can finally transfer the nucleus into the mass center of the spheroid within $\sim 100 \mathrm{Myr}(T=561 \mathrm{Myr})$. Thus a stellar galactic nucleus composed of new stars can be formed from merging between massive stellar and gaseous clumps in the central region of the spheroid.

Figure 3 shows the time evolution of the star formation rate in the fiducial model M1 with feedback effects of supernovae and in the model M2 without. The peak star formation rate $\left(0.6 \mathrm{M}_{\odot} \mathrm{yr}^{-1}\right)$ in $\mathrm{M} 2$ is higher than that of M1 by a factor of about three, which suggests self-regulation of star formation by supernovae feedback is a key determinant for the star formation histories in the nuclear regions of galaxies. The star formation rate in M1 continues to be relatively high $\left(\sim 0.2 \mathrm{M}_{\odot} \mathrm{yr}^{-1}\right)$ during the mutual interaction of massive gas-rich clumps $(100<T<400 \mathrm{Myr})$. The star formation rate becomes lower $\left(<0.1 \mathrm{M}_{\odot} \mathrm{yr}^{-1}\right)$ after the formation of a single nucleus via merging of the clumps ( $T>400 \mathrm{Myr})$.

As shown in Figure 4, the total stellar mass of new stars that are the building blocks of the stellar nucleus can gradually increase over $400 \mathrm{Myr}(200<T<600 \mathrm{Myr})$. The total mass of old stars, on the other hand, can be kept nearly constant during the nucleus formation, which means that the nuclear region $(R<100 \mathrm{pc})$ becomes progressively more dominated by the new stellar population as time passes. For example, the mass fraction of new stars is only $\sim 0.05$ at $T=200 \mathrm{Myr}$ whereas it is $\sim 0.5$ at $T=686 \mathrm{Myr}$. Given the fact that the mass-to-light ratio of young stellar populations with ages less than $1 \mathrm{Gyr}$ is much smaller than that of old ones with ages of $10 \mathrm{Gyr}$ for the adopted IMF in the present study, the derived high mass fraction of new stars implies that the spectrophotometric properties of the nuclear region in M1 can be largely determined 


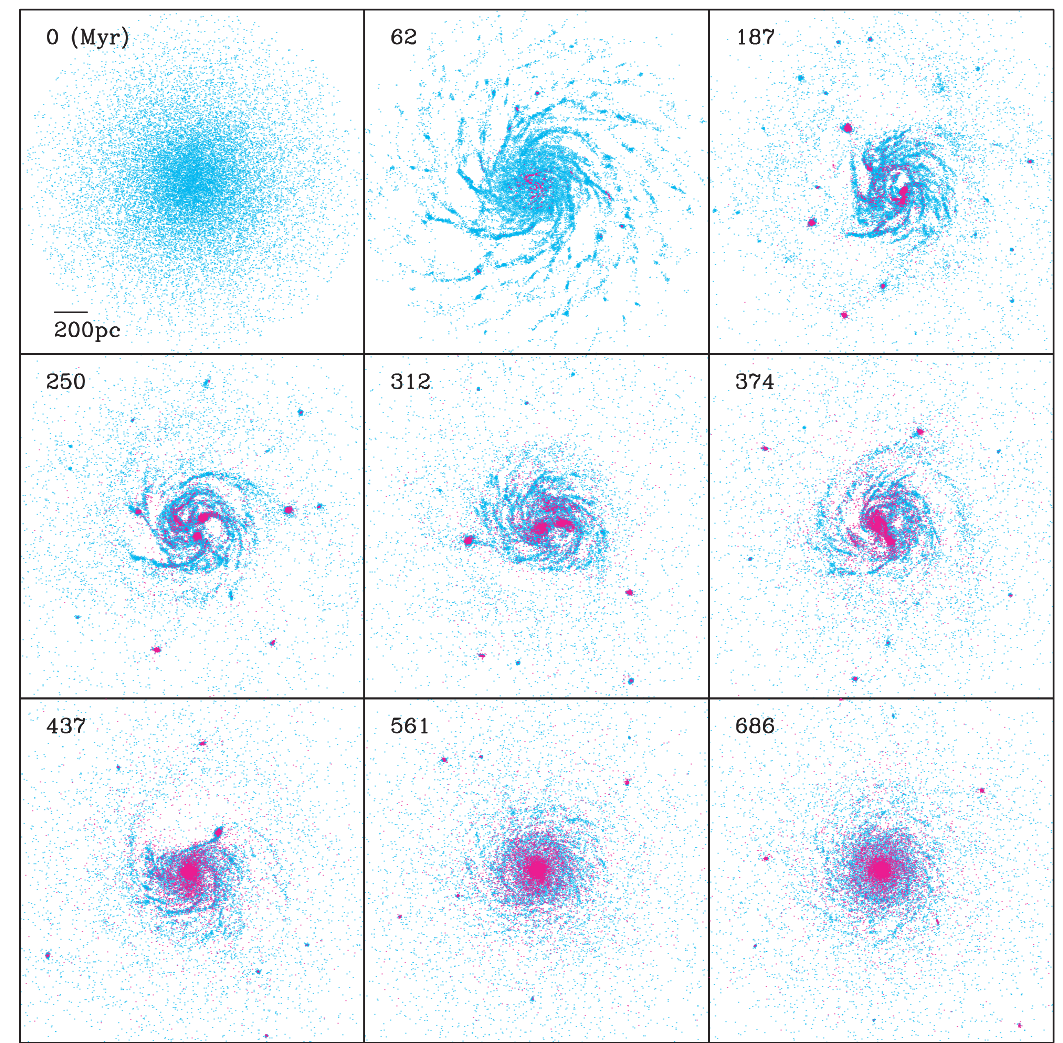

Figure 1 Morphological evolution of gas (cyan) and new stars (magenta) in the fiducial model M1 projected onto the $x-y$ plane. The time $T$ shown in the upper left corner of each panel represents the time that has elapsed since the simulation starts. One frame measures $2 \mathrm{kpc}$ on a side.

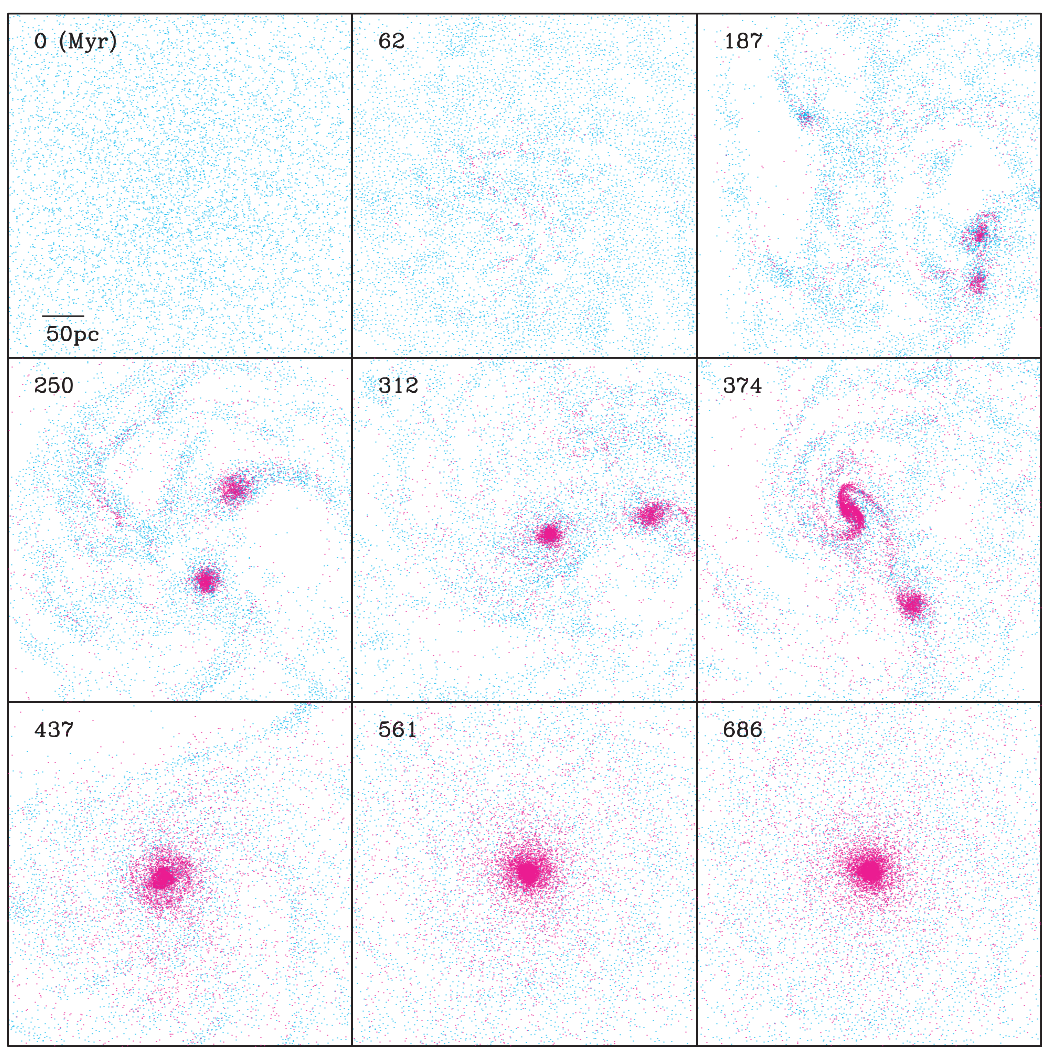

Figure 2 The same as Figure 1 but for a smaller scale. One frame measures $400 \mathrm{pc}$ on a side. 


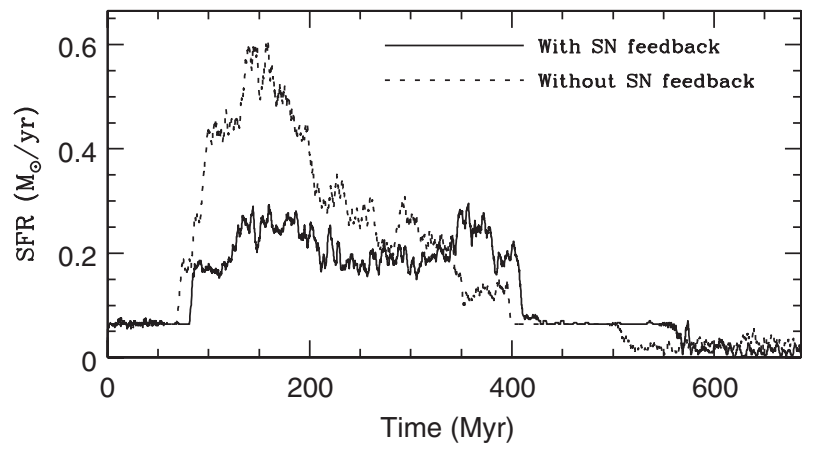

Figure 3 Time evolution of star formation rates (SFRs) for the fiducial model M1 with supernova feedback effects (solid) and for the model M2 without (dotted).

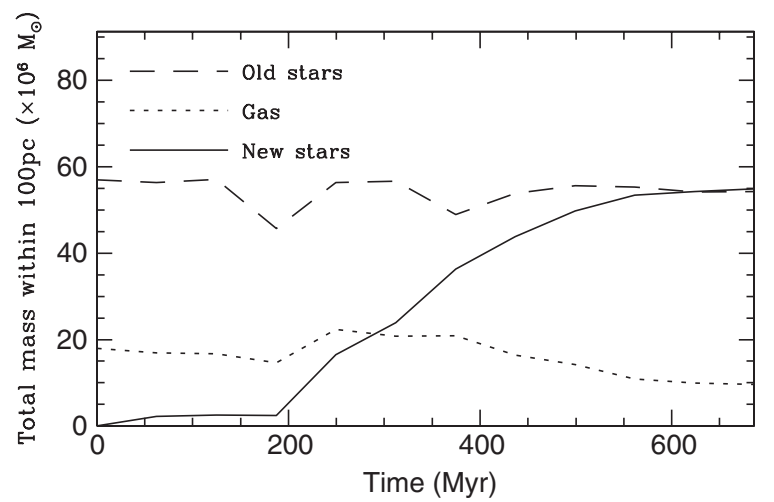

Figure 4 Time evolution of the total mass within the central $100 \mathrm{pc}$ for old stars (dashed), gas (dotted), and new stars (solid) in the fiducial model M1.

by the new populations rather than by the background old ones.

\subsubsection{Structure}

Figure 5 shows how the radial density profiles of stellar components (i.e. old and new stars) evolve with time in M1. As numerous clumps composed of gas and new stars are formed and then merged with one another, the projected density of new stars can gradually become higher ( $T=250 \mathrm{Myr}$ ). It should be stressed here that the central density of new stars within the central $10-100 \mathrm{pc}$ is not high at all in the early evolutionary stage of stellar nucleus formation (e.g. $T=250 \mathrm{Myr}$ ). This is essentially because massive clumps can be initially formed outside the nuclear region of the galaxy. The central stellar density of the galaxy finally becomes as high as $7 \times 10^{4} \mathrm{M}_{\odot} \mathrm{pc}^{-2}$ after the merging of massive clumps and the resultant single stellar nucleus formation ( $T=686 \mathrm{Myr})$. Initially the flat radial distribution of old stars due to the adopted exponential profile of the projected density distribution does not change during the stellar nucleus formation.

By combining the above result in Figure 5 with the stellar-population synthesis code adopted in the present study (i.e. Vazdekis et al. 1996), we can show what the radial profile looks like in the $B$-band. Figure 6 demonstrates that the radial light profile depends strongly on the ages of the new stellar populations, in the sense that the

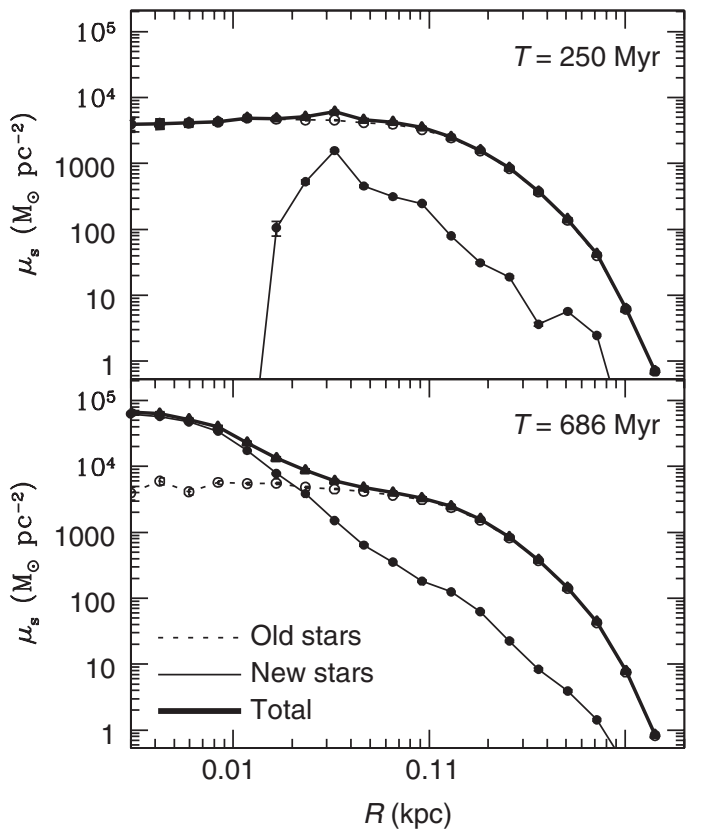

Figure 5 The projected radial density profiles $\left(\mu_{\mathrm{s}}\right)$ for old stars (dotted), new stars (thin solid), and total (i.e. old and new stars, thick solid) at $T=250 \mathrm{Myr}$ (upper) and $T=686 \mathrm{Myr}$ (lower) in the fiducial model M1.

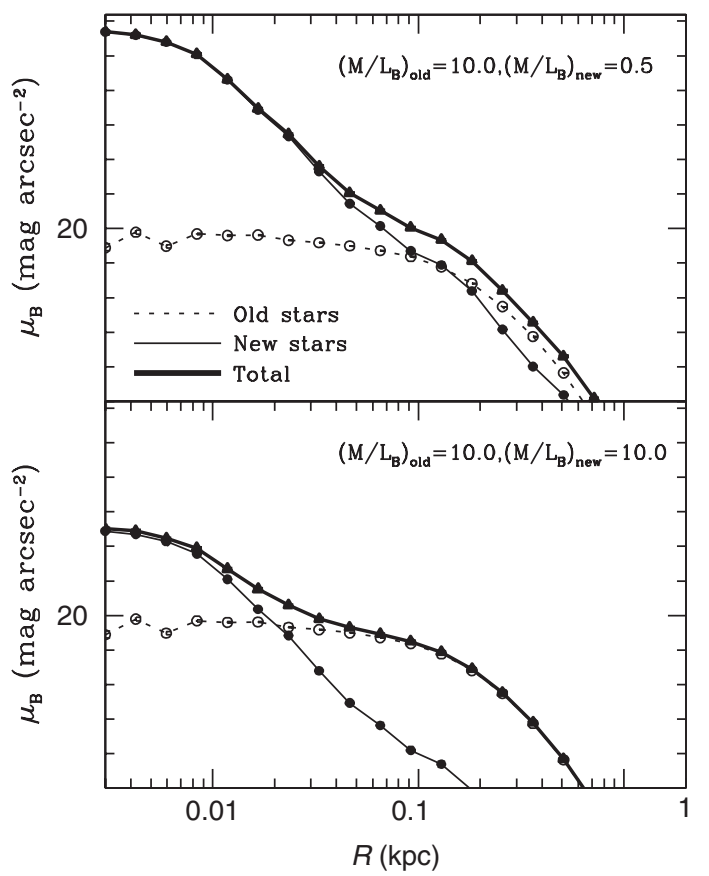

Figure $6 \quad B$-band surface-brightness profiles $\left(\mu_{\mathrm{B}}\right)$ for old stars (dotted), new stars (thin solid), and total (i.e. old + new stars, thick solid) at $T=686 \mathrm{Myr}$ in the fiducial model M1 with $\left(M / L_{\mathrm{B}}\right)_{\text {old }}=10$ and $\left(M / L_{\mathrm{B}}\right)_{\text {new }}=0.5$ (upper) and in that with $\left(M / L_{\mathrm{B}}\right)_{\text {old }}=10$ and $\left(M / L_{\mathrm{B}}\right)_{\text {new }}=10$ (lower). The results in the upper frame corresponds to the epoch when the SGN is young $(\sim 0.5 \mathrm{Gyr})$.

stellar nucleus appears to be more pronounced at their younger ages owing to the smaller mass-to-light ratio (i.e. $\left(M / L_{\mathrm{B}}\right)_{\text {new }}=0.5$ for a SSP with $\left.0.5 \mathrm{Gyr}\right)$. The difference in the central $\mu_{\mathrm{B}}$ between the young nucleus and the background old stars is $\sim 3$ mag for the model 

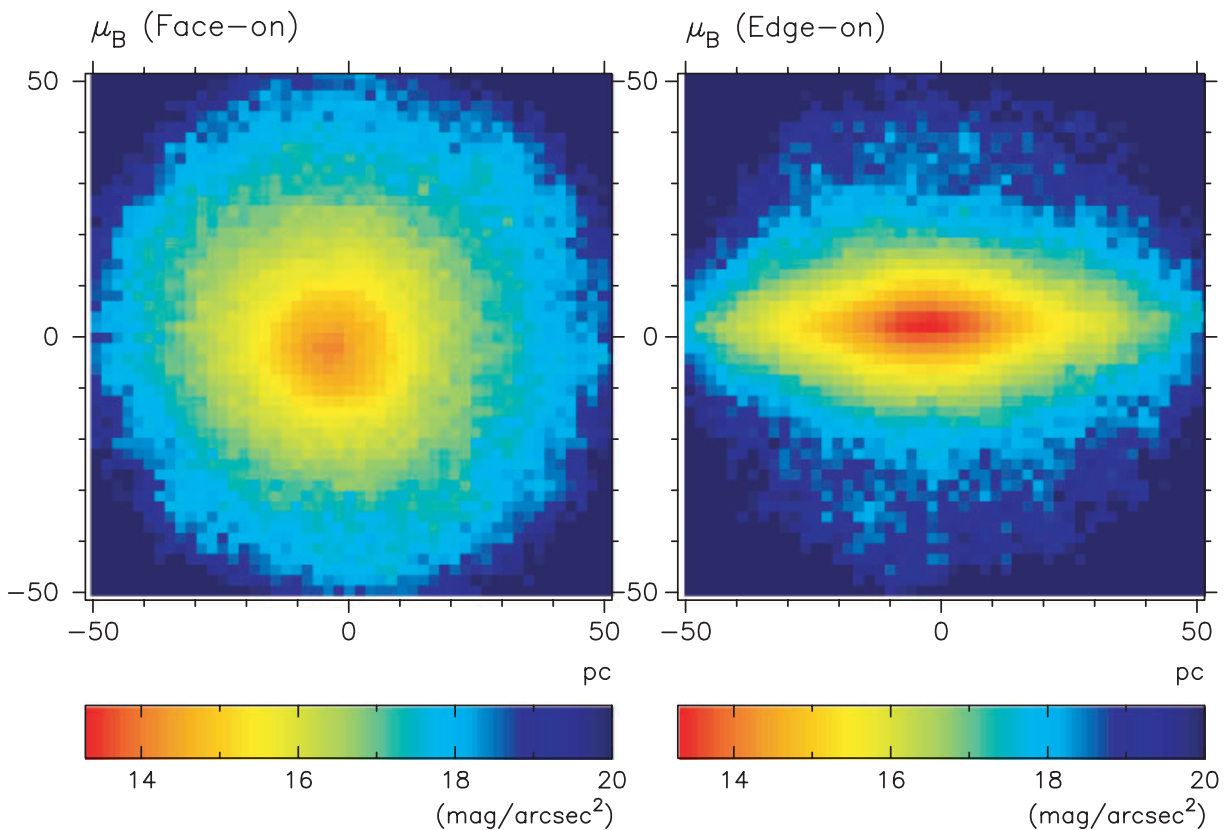

Figure 7 Two dimensional (2D) distributions of $\mu_{\mathrm{B}}$ at $T=686 \mathrm{Myr}$ for the face-on view (left) and for the edge-on one (right) in the fiducial model M1 with $\left(M / L_{\mathrm{B}}\right)_{\text {old }}=10$ and $\left(M / L_{\mathrm{B}}\right)_{\text {new }}=10$.

with $\left(M / L_{\mathrm{B}}\right)_{\text {old }}=10$ and $\left(M / L_{\mathrm{B}}\right)_{\text {new }}=10$ (i.e. a SSP with $10 \mathrm{Gyr}$ ) whereas it is $\sim 6 \mathrm{mag}$ for the model with $\left(M / L_{\mathrm{B}}\right)_{\text {old }}=10$ and $\left(M / L_{\mathrm{B}}\right)_{\text {new }}=0.5$ (i.e. a SSP with $0.5 \mathrm{Gyr})$. These results indicate that the surface-brightness profiles of nucleated galaxies depend strongly on when their nuclei were formed.

Figure 7 shows the face-on (projected onto the $x-y$ plane) and the edge-on (the $x-z$ ) views of the stellar nucleus of the galaxy at $T=686 \mathrm{Myr}$ in M1. In order to clearly show the mass distribution of new stars, only stellar particles within the central $50 \mathrm{pc}$ are used to create the $2 \mathrm{D}$ distribution of $\mu_{\mathrm{B}}$. The stellar nucleus appears to be a 'nuclear star cluster' owing to its compact, round shape in the face-on view whereas it is very flattened and looks like a 'disky elliptical' (or S0) galaxy. The stellar nucleus formed from dissipative merging between clumps composed of gas and stars can have a significant amount of rotation owing to the conversion of orbital angular momentum of the clumps into the intrinsic angular momentum of the nucleus. This rotationally flattened stellar nucleus is one of the characteristics of dissipative nucleus formation in a gas disk embedded by an old stellar spheroid.

\subsubsection{Kinematics}

Figure 8 shows the radial profiles of the rotational velocity $\left(V_{\text {rot }}\right)$ and the velocity dispersion $(\sigma)$ estimated separately for the old stars and the new stars at $T=686 \mathrm{Myr}$ in M1. It is clear from this figure that the stellar nucleus composed mostly of new stars can show a significant amount of rotation $\left(20-50 \mathrm{~km} \mathrm{~s}^{-1}\right.$ within the central $100 \mathrm{pc}$ ). It is remarkable that the new stars show a radially increasing profile of $\sigma$ with the central velocity

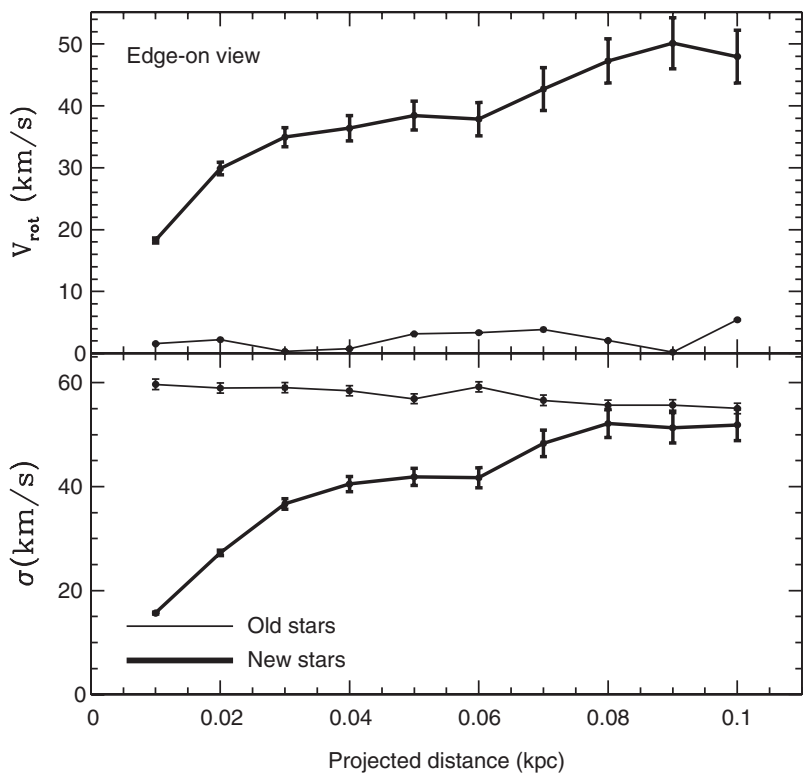

Figure 8 Radial profiles of rotational velocity (upper) and velocity dispersion (lower) for old stars (thin solid) and new ones (thick solid) at $T=686 \mathrm{Myr}$ for the edge-on view (i.e. projected onto the $x-z$ plane) in the fiducial model M1.

dispersion $\left(\sigma_{0}\right)$ of $\sim 15 \mathrm{~km} \mathrm{~s}^{-1}$ whereas the old stars show the nearly constant profile with $\sigma_{0}$ of $\sim 60 \mathrm{~km} \mathrm{~s}^{-1}$. The essential reason for the lower $\sigma_{0}$ in the new stars is that the stellar nucleus, which is formed from dissipative merging of clumps, can be strongly self-gravitating so that $\sigma_{0}$ is determined by the nucleus itself (i.e. by its smaller mass of $\sim 0.05$ in our simulation units). The radially increasing profile of $\sigma$ in the new stars is derived for the stellar kinematics seen from the face-on view. 


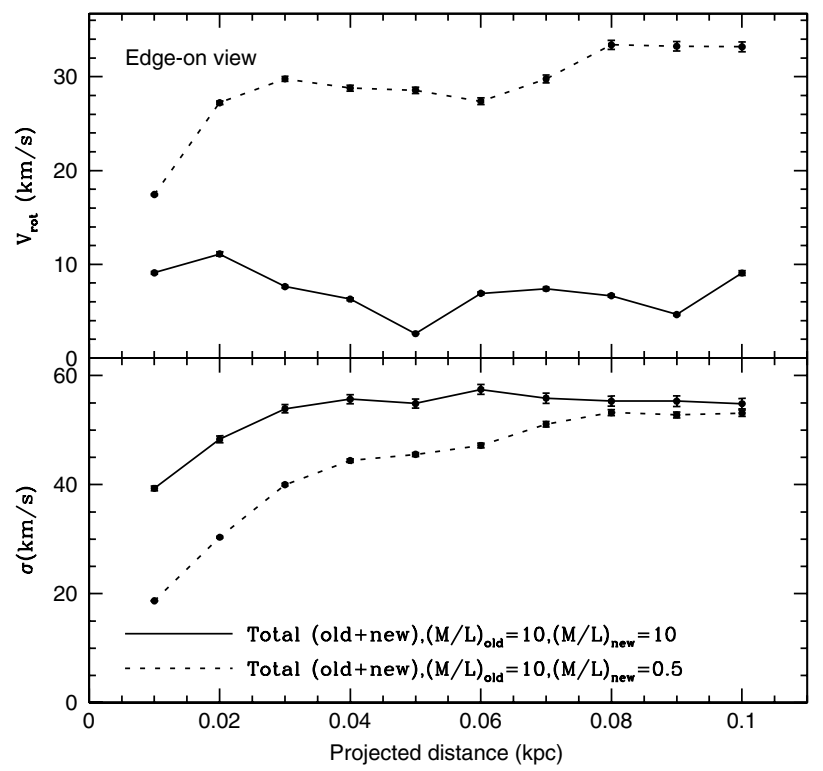

Figure 9 Luminosity-weighted radial profiles of rotational velocity (upper) and velocity dispersion (lower) of stars (i.e. old and new stars) at $T=686 \mathrm{Myr}$ for the edge-on view in the fiducial model M1 with $\left(M / L_{\mathrm{B}}\right)_{\text {old }}=10$ and $\left(M / L_{\mathrm{B}}\right)_{\text {new }}=10$ (solid) and that with $\left(M / L_{\mathrm{B}}\right)_{\text {old }}=10$ and $\left(M / L_{\mathrm{B}}\right)_{\text {new }}=0.5$ (dotted)

By combining the above result in Figure 8 with the stellar population synthesis code adopted in the present study (i.e. Vazdekis et al. 1996), we can calculate the luminosityweighted $V_{\text {rot }}$ and $\sigma$ profiles, which can be compared directly with the corresponding observations derived from spectroscopic studies of stellar kinematics. Figure 9 shows that radially increasing profiles of $\sigma$ can be seen for the two representative $M / L$ models even if we take a luminosityweighted average of $\sigma$ of stars (i.e. both old and new stars) at each radial bin. This result suggests that nucleated galaxies can have radially increasing $\sigma$ profiles, if their stellar galactic nuclei are formed from dissipative merging of massive clumps within the central $1 \mathrm{kpc}$ of galaxies. The rotation profile $\left(V_{\text {rot }}\right)$ depends strongly on the ages of the new stars; the central region of the nucleated galaxy $R<100$ pc can show a significant amount of rotation only when the stellar nucleus is young $(<1 \mathrm{Gyr})$.

\subsubsection{Chemical Properties}

Figure 10 shows the age-metallicity relation of the new stars that are located within the central $50 \mathrm{pc}$ (thus within the nucleus) of the galaxy at $T=686 \mathrm{Myr}$ in M1. The mean age and metallicity of this stellar nucleus are estimated as $3.7 \times 10^{8}\left(=10^{8.57}\right)$ yr and as -0.29 in $[\mathrm{Fe} / \mathrm{H}]$, respectively. The final metallicity of -0.29 is significantly higher than the initial one $\left([\mathrm{Fe} / \mathrm{H}]_{\mathrm{i}}=-0.44\right)$ for the chemical yield of 0.005 and $[\mathrm{Fe} / \mathrm{H}]_{\mathrm{i}}=-0.44$, which means that the stellar nucleus is more metal-rich and younger than the background stellar (spheroidal) components. Figure 10 clearly indicates that the stellar nucleus is composed of stars with different ages and metallicities: the ages of the new stars range from to $6.3 \times 10^{6}\left(=10^{6.8}\right)$ yr to $6.8 \times 10^{8}$ $\left(=10^{8.8}\right) \mathrm{yr}$, whereas the metallicities range from -0.44 to

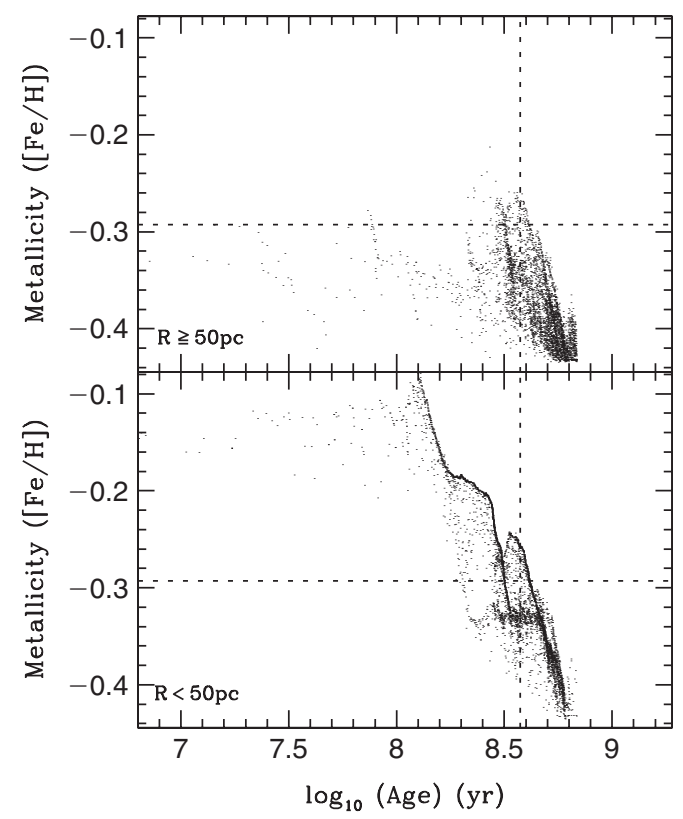

Figure 10 Distribution of new stars on the age-metallicity map at $T=686 \mathrm{Myr}$ in the fiducial model M1 with the initial gaseous metallicity $[\mathrm{Fe} / \mathrm{H}]_{\mathrm{i}}$ of -0.44 . Here new stars with distances from the center $(R)$ being equal to or larger than $50 \mathrm{pc}$ and those with $R<50$ pc (thus those regarded as being in the SGN) are shown in the upper and lower panels, respectively.

-0.077 in $[\mathrm{Fe} / \mathrm{H}]$. This wider ranges of ages and metallicities reflect the fact that the stellar nucleus can form from merging between different clumps, which have different star-formation and chemical-evolution histories.

It is clear from Figure 10 that the more metalrich stars are more likely to be younger. This derived age-metallicity relation between stars of the nucleus reflect the fact that younger stars can be formed from more metal-rich gas within each of massive gas-rich clumps. The stellar nucleus itself does not show any significant metallicity gradients of the stellar population within the central $50 \mathrm{pc}$, which is due probably to the efficient dynamical mixing of stars during merging of clumps. The derived young, metal-rich stellar population of the nucleus implies that the more metal-rich nucleus (compared to its host spheroid) does not necessarily mean a redder nucleus, because of its younger nucleus. We will discuss later (Section 4) the observed color difference between stellar nuclei and their host galaxies based on this result.

\subsection{Parameter Dependences}

Although the numerical results of structure, kinematics, and chemical properties of SGN are similar to one another between the fiducial model M1 and other models, these depend primarily on (a) the spheroidal mass $\left(M_{\mathrm{sph}}\right)$, (b) the initial gas mass fraction $\left(f_{\mathrm{g}}\right)$, (c) whether galaxies are LSBs or HSBs, and (d) whether the adopted IMF is a top-heavy one. Since the formation processes, the shapes and the kinematics of the simulated SGN do not depend so strongly on the above parameters, we focus on the 


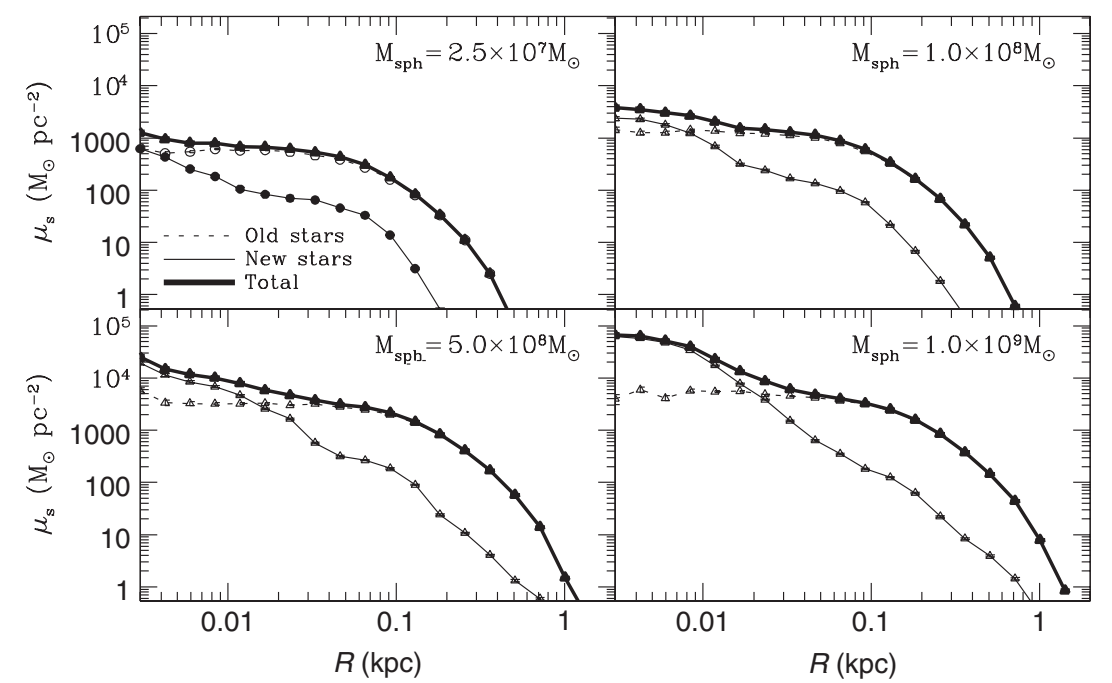

Figure 11 Dependences of $\mu_{\mathrm{s}}$ on $M_{\mathrm{sph}}$. Here the results of the model M11 with $M_{\mathrm{sph}}=2.5 \times 10^{7} \mathrm{M}_{\odot}$ (upper left), M12 with $M_{\mathrm{sph}}=10^{8} \mathrm{M}_{\odot}$ (upper right), M13 with $M_{\mathrm{sph}}=5.0 \times 10^{8} \mathrm{M}_{\odot}$ (lower left), and M1 with $M_{\mathrm{sph}}=10^{9} \mathrm{M}_{\odot}$ (lower right) are shown. Old stars, new ones, and total (i.e. old + new stars) are shown by dotted, thin solid, and thick solid lines, respectively, in each panel.

parameter dependences of the simulated structural properties of SGN.

\subsection{1 $M_{\mathrm{sph}}$}

Figure 11 shows the projected radial density profiles $\left(\mu_{\mathrm{s}}\right)$ of stellar components in the models (M1, M11, M12, and M13) with different masses of the spheroids $\left(2.5 \times 10^{7} \mathrm{M}_{\odot} \leq M_{\text {sph }} \leq 1.0 \times 10^{9} \mathrm{M}_{\odot}\right)$. Although these models appear to show the formation of SGN within the central $10 \mathrm{pc}$, the central densities and the masses of the SGN are diversely different from with one another. It is clear from Figure 11 that the SGN are more likely to show higher surface brightness in more massive spheroids. Furthermore, SGN are found to be more massive (for the central $100 \mathrm{pc}$ ) in more massive spheroids. This thus indicates that more massive SGN can have higher central stellar densities, which is strikingly similar to the observed scaling relation of the Galactic GCs (e.g. Djorgovski et al. 1997). The essential reason for the derived more pronounced SGN in more massive galaxies is that supernovae feedback can more strongly suppress (a) efficient star formation and (b) formation of massive gas clumps (which can be building blocks of SGN) in less massive galaxies with shallower gravitational potentials.

As shown in Figure 11, the central 'bumps' or 'cusps' in the $\mu_{\mathrm{s}}$ profile in lower mass models with $M_{\text {sph }} \leq 10^{8} \mathrm{M}_{\odot}$ are less likely to be regarded as SGN literally in observations, in particular, when the nuclear bumps become old (i.e. larger $M / L$ ). This result implies that massive and pronounced SGN are less likely to be formed in less massive spheroids. It is also found that the time scale of SGN formation is longer for less massive spheroids (e.g. less than $0.5 \mathrm{Gyr}$ for $M_{\text {sph }} \sim 10^{9} \mathrm{M}_{\odot}$ and a few Gyr for $\left.M_{\mathrm{sph}} \sim 10^{7}-10^{8} \mathrm{M}_{\odot}\right)$, because of longer time scales of clumps spiraling in due to dynamical friction in less massive galaxies. This result implies that if less massive spheroids have SGN, SGN are likely to be younger (thus possibly bluer) than those in more massive spheroids. Furthermore more massive spheroids can contain more metal-rich SGN, which suggests that there can be a positive correlation between the metallicities of SGN and those of their host galaxies owing to the observed mass-metallicity (or luminositymetallicity) relations of spheroids (e.g. Mould 1984).

\section{$3.2 .2 f_{\mathrm{g}}$}

Figure 12 shows how $\mu_{\mathrm{s}}$ profiles depend on the initial gas mass fraction $\left(f_{\mathrm{g}}\right)$ for the models with different $f_{\mathrm{g}}$ (M1, M5, M6, and M7). It is clear from this Figure 12 that (a) spheroids with smaller $f_{\mathrm{g}}(\leq 0.1)$ cannot develop SGN within a few Gyr and (b) the central $\mu_{\mathrm{s}}$ of SGN is higher in the model with larger $f_{\mathrm{g}}$. The origin of these dependences is closely associated with the fact that the total amount of gaseous dissipation, which is larger for the models with larger $f_{\mathrm{g}}$, can be a key determinant for the development of SGN in the central gas-rich regions of spheroids. It is also found that the total masses of new stars within the central $100 \mathrm{pc}$ are larger in the models with larger $f_{\mathrm{g}}$. These results imply that more massive and pronounced SGN are more likely to be formed in more gas-rich galaxies (i.e. later Hubble-types). We confirm this $f_{\mathrm{g}}$ dependence for the model with $M_{\mathrm{sph}}=10^{8} \mathrm{M}_{\odot}(\mathrm{M} 12$, M14, M15, and M16).

\subsubsection{Surface Brightness}

As Figure 13 reveals, showing $\mu_{\mathrm{s}}$ profiles for the LSB model M3, the fiducial model M1 and the HSB model M4, the central $\mu_{\mathrm{s}}$ of SGN are higher for the models with higher surface brightness of their spheroids. This is because a larger amount of gaseous dissipation and thus more efficient transfer of massive clumps into the nuclear regions of spheroids is possible in the models with higher surfacebrightness spheroids and thus with higher gas densities. 


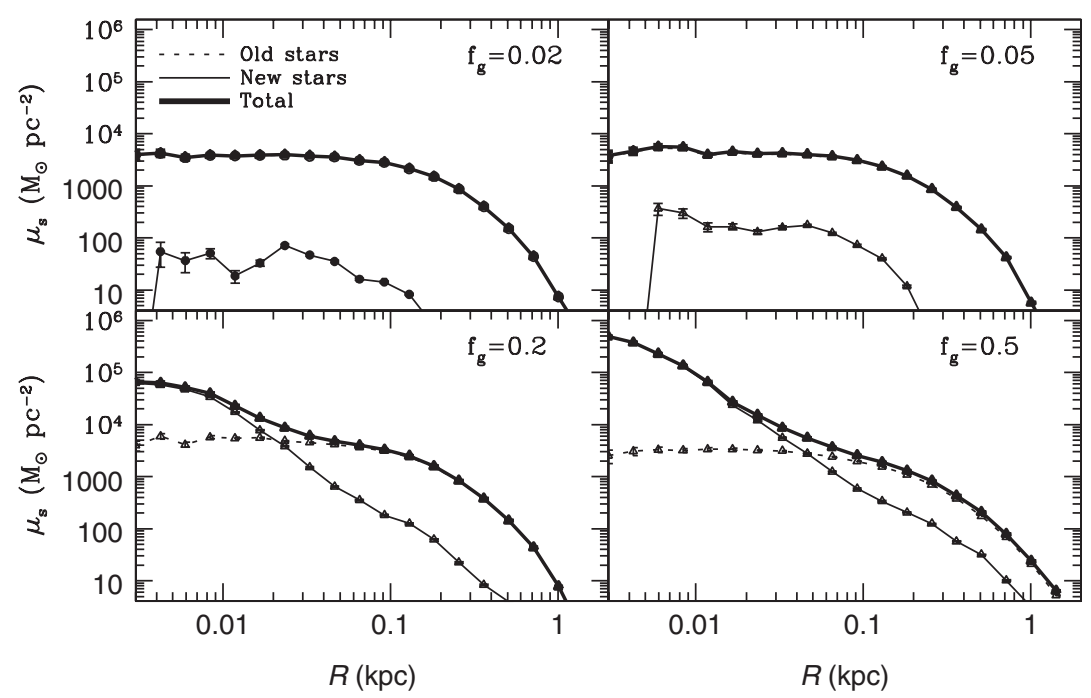

Figure 12 Dependences of $\mu_{\mathrm{s}}$ on $f_{\mathrm{g}}$. Here the results of the model M5 with $f_{\mathrm{g}}=0.02$ (upper left), M6 with $f_{\mathrm{g}}=0.1$ (upper right), M1 with $f_{\mathrm{g}}=0.2$ (lower left), and M7 with $f_{\mathrm{g}}=0.5$ (lower right) are shown. Old stars, new ones, and total (i.e. old + new stars) are shown by dotted, thin solid, and thick solid lines, respectively, in each panel.

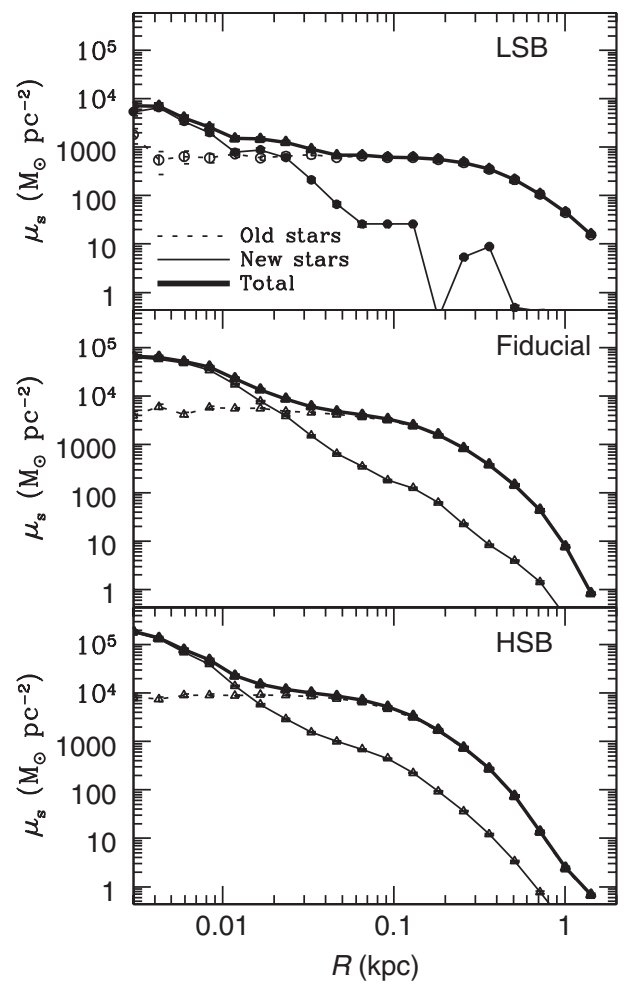

Figure 13 Dependences of $\mu_{\mathrm{s}}$ on the initial surface brightness of spheroids. Here the results of the LSB model M3 (top), the fiducial one M1 (middle), and HSB one M4 (bottom) are shown. Old stars, new ones, and total (i.e. old + new stars) are shown by dotted, thin solid, and thick solid lines, respectively, in each panel.

This result indicates that there can be a positive correlation between the surface brightness of SGN and those of their host galaxies. The mass fraction of SGN, however does not depend so strongly on surface brightness of their spheroids: It is roughly 0.05 for the three models. This result implies that LSBs can contain SGN, though the central densities of the SGN can be lower than those of HSBs.

\subsubsection{Massive Black Holes}

It is found that there are no significant differences in structural, kinematic and chemical properties between M1 (the fiducial model), M8 with $M_{\mathrm{mbh}}=0.006 M_{\mathrm{sph}}$ and M9 with $M_{\mathrm{mbh}}=0.018 M_{\mathrm{sph}}$. This result implies that SGN formation in a spheroid is not influenced by the nuclear $\mathrm{MBH}$, the total masses of which are much less than gas disks: selfgravity of gas is much more dynamically important than the gravitational influence of the central point mass. All the three models show small central velocity dispersions $\left(<20 \mathrm{~km} \mathrm{~s}^{-1}\right)$ in new stars and thus are not so consistent with observations for some dE,Ns. We will discuss this point in Section 4.2.

\subsubsection{IMF}

SGN formation is found to be strongly suppressed in the model M10 with the top-heavy IMF (i.e. 2.5 times more $\mathrm{SNe}$ explosions compared with the fiducial model), because the formation of massive clumps, which are 'building blocks' of the SGN, is suppressed owing to the stronger feedback effects of supernovae in this model. A few clumps continue to orbit the mass center of the spheroid in the top-heavy IMF model and, consequently, no single nucleus with the central $\mu_{\mathrm{s}}>10^{4} \mathrm{M}_{\odot} \mathrm{pc}^{-2}$ can be formed in this model within $\sim 1$ Gyr. These results suggest that IMF is a key parameter that can control SGN formation. Considering that IMF can be top-heavy in the early universe and in star-forming regions with very low metallicities (e.g. Larson 1998), the above results imply that SGN formation in less luminous dwarfs that were formed at higher redshifts and had lower metallicities could be less likely.

It is important to check how the results are changed if newly born stars are dominated by low-mass stars, as 
observed in the solar neighborhood. In the model M18 with $m_{\mathrm{u}}=10 \mathrm{M}_{\odot}$ (i.e. smaller number of Type-II SNe) the mass fraction of new stars is larger by a factor of 1.3 than in the fiducial model (M1), whereas the maximum star formation rate in nuclear regions is higher by a factor of 1.7 than in M1. Consequently, the mass of SGN in this model is larger by a factor of 1.1 than that in M1. The mass of SGN in the model M19 is larger by a factor of 1.2 than that in M1. The origin of the derived larger masses of SGN in these two models is closely associated with weaker feedback effects of Type-II SNe.

\subsubsection{Miscellaneous}

Models with different gravitational softening lengths $\left(\epsilon_{\mathrm{g}}\right)$ and different initializations of gas disks were investigated to check the robustness of the present results. The results of the models are summarized as follows. Firstly formation processes of SGN (i.e. merging of smaller clumps composed of gas and stars) do not depend on $\epsilon_{\mathrm{g}}$ for a reasonable range of $\epsilon_{\mathrm{g}}$. However the total masses of SGN can depend weakly on $\epsilon_{\mathrm{g}}$. For example, the total mass of SGN in the model M20 with $\epsilon_{\mathrm{g}} 0.54$ times the softening length of the fiducial model (M1) is larger by a factor of 1.1 than that of SGN in M1. The larger mass of SGN in M20 can be due to the fact that more massive clumps, that can finally merge to form SGN, can be formed from local instabilities of the gas disk owing to the stronger gravity in M20 with smaller $\epsilon_{\mathrm{g}}$.

Secondly, formation processes of SGN are essentially similar between models with different initializations of gas disks. However the initial locations of big clumps formed in gas disks are different between different models so that the final merging processes of these clumps (e.g. the epoch of clump merging and the number of clumps responsible for SGN formation) are slightly different between different models. Consequently the total masses of new stars and those of SGN can be slightly different between models with different initializations of gas disks. For example, the mass of new stars in the model M21 is smaller by a factor of 1.1 than that of the fiducial model whereas the SGN mass is smaller by a factor of 1.2 than that of the fiducial model.

\section{Discussion}

\subsection{Predictions from a Dissipative Nucleus- Formation Scenario}

We have investigated structural, kinematic, and chemical properties of SGN formed in a dissipative way within the central gas disks embedded in old stellar spheroids. We have found that (a) SGN show great diversity in their physical properties depending on their host masses and gas mass fraction and (b) they show some correlations between the physical properties of the SGN and those of their host spheroids. The derived diversity and uniformity in the SGN properties can be tested against ongoing and future observations. We briefly summarize these as follows.

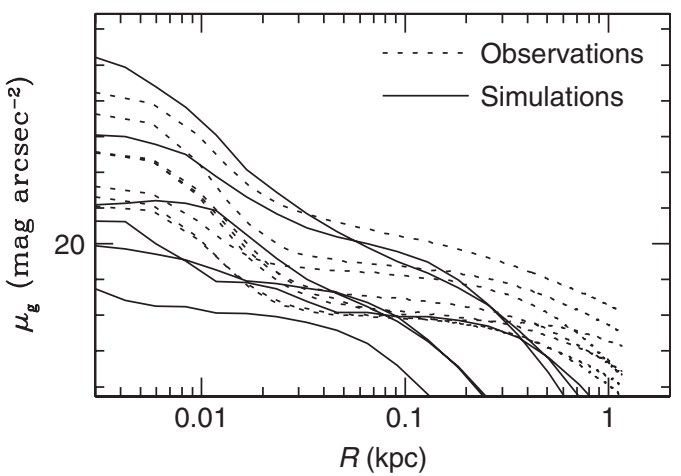

Figure 14 Comparison of the simulated $\mu_{\mathrm{g}}$ profiles of nucleated spheroids (solid) with the observed one of eight dE,Ns in the Virgo cluster of galaxies (dotted). Here the Virgo distance modulus of 30.92 is adopted and the HST observational results of VCC 0009, 0033, $1075,1087,1185,1261,1355$, and 1489 derived by de Propris et al. (2005) are shown. $B-g=0.54$ mag is adopted for the simulated $\mu_{\mathrm{g}}$ profiles and the results of the representative models, M1, M3, M7, M11, M12, and M16 are shown.

\subsubsection{Structure}

1. The radial surface-brightness profiles of nucleated spheroids can be remarkably diverse (see Figure 14 for the comparison between the simulations and the observations). This is due to the fact that the formation processes of SGN depends strongly on $M_{\mathrm{sph}}, f_{\mathrm{g}}$, and the surface brightness of the spheroids.

2. The intrinsic shapes of SGN are rather flattened, irrespective of model parameters. More massive SGN are more likely to have higher central stellar densities.

3. More-massive spheroids are more likely to develop SGN and have more-massive, higher-density SGN.

4. More gas-rich, higher-surface-brightness spheroids are more likely to develop more-massive SGN.

\subsubsection{Kinematics}

1. Irrespective of model parameters, nucleated spheroids can have radially increasing $\sigma$ profiles (i.e. smaller velocity dispersions in the inner regions).

2. SGN with flattened shapes are dynamically supported by rotation $\left(V_{\text {rot }} / \sigma_{0}>1\right)$ rather than by anisotropic velocity dispersion.

\subsubsection{Abundance}

1. Metallicities of SGN are higher than those of their host galaxies and accordingly SGN can be redder than their host galaxies after significant aging of the nuclear stellar populations.

2. More-massive spheroids can have more metal-rich SGN. Less-massive spheroids can have younger SGN owing to the longer time scales of SGN formation.

\subsubsection{Correlations between Physical Properties of Stellar Galactic Nuclei and Their Host Galaxies}

1. The total mass of SGN $\left(M_{\text {nuc }}\right)$ within $50 \mathrm{pc}$ (5\% of an initial gas disk size) in units of $10^{9} \mathrm{M}_{\odot}$ is correlated with the total mass of spheroids $\left(M_{\mathrm{sph}}\right)$ in units 
of $10^{9} \mathrm{M}_{\odot}$ and this correlation can be described as:

$$
M_{\mathrm{nuc}}=0.046 \times M_{\mathrm{sph}}-0.0030 .
$$

Here we apply the least-squares fit to the results of the models (M1, M11, M12, and M13) that have different $M_{\text {sph }}$ and clearly show SGN formation. If we make a least-squares fit to the $\log _{10} M_{\mathrm{nuc}}-\log _{10} M_{\mathrm{sph}}$ relation, we can derive the following:

$$
\log _{10} M_{\text {nuc }}=1.1 \times \log _{10} M_{\mathrm{sph}}-2.5 \text {. }
$$

2. The mass fraction of $\operatorname{SGN}\left(f_{\text {nuc }}\right)$ in nucleated spheroids is correlated with $M_{\text {sph }}$ (in units of $10^{9} \mathrm{M}_{\odot}$ ) and the correlation can be described as:

$$
f_{\text {nuc }}=0.021 \times M_{\mathrm{sph}}+0.023 .
$$

3. The half-mass radii ( $R_{\mathrm{e}, \text { nuc }}$ in units of $\mathrm{pc}$ ) of new stars within the central $100 \mathrm{pc}$ of spheroids is correlated with $M_{\text {nuc }}$ (in units of $10^{6} \mathrm{M}_{\odot}$ ) and this correlation can be described as:

$$
R_{\mathrm{e}, \mathrm{nuc}}=-2.78 \times M_{\mathrm{nuc}}+26.2 \text {. }
$$

Although this $R_{\mathrm{e}, \text { nuc }}$ can be possibly regarded as the half-mass radii of SGN, these values should be carefully compared with the observations (e.g. Böker et al. 2004), for which $R_{\mathrm{e}, \text { nuc }}$ is estimated for the nuclear stars (not for the entire stellar components in the nucleated galaxies).

Only some of these predictions can be compared with observations (as discussed later in Section 4.2), though a growing number of observations on physical properties of SGN have now been accumulated (e.g. Böker et al. 2004). Statistical studies of the ellipticity distribution of apparent shapes of SGN can provide some valuable information on the intrinsic shapes of SGN, as has been demonstrated for studies of elliptical galaxies (e.g. Lambas, Maddox \& Loveday 1992). Also spectroscopic determination of mean metallicities of SGN is also doubtlessly worthwhile, because it enables us to discuss the predicted correlation between metallicities of SGN and those of their host galaxies.

\subsection{Comparison with Observations}

\subsubsection{Correlations between $L_{\mathrm{nuc}}$ and $L_{\mathrm{bul}}$}

Recent HST observations of SGN in spiral galaxies with different Hubble types have found the following two correlations between the physical properties of SGN and those of their host galaxies (e.g. Carollo et al. 1988; Balcells et al. 2003; Böker et al. 2004). The first is that more luminous galaxies can have more luminous nuclei: Balcells et al. (2003) found that $L_{\text {nuc }} \propto L_{\text {bul }}^{0.50 \pm 0.14}$, where $L_{\text {nuc }}$ and $L_{\text {bul }}$ are the $K$-band luminosities of the point nuclear sources (SGN) and those of their host bulges. Böker et al. (2004) also revealed that $L_{\text {nuc }} \propto L_{\text {disk }}^{0.78}$, where $L_{\text {disk }}$ is the total luminosities of the disks of late-type galaxies with
SGN. The second is that galaxies with higher central surface brightness can have more luminous SGN for late-type galaxies with $M_{\mathrm{B}}$ ranging from -16 to -20.5 mag (Böker et al. 2004).

The first correlation (i.e. the value of the slope) is qualitatively consistent with the present theoretical predictions shown in the equations (14) and (15), though the slopes $\alpha$ in the relation $L_{\text {nuc }} \propto L_{\text {bul }}^{\alpha}$ are slightly different between the simulations and the observations. The second correlation is also consistent qualitatively with the present results, which show that galactic spheroids with higher surface brightness can have more pronounced SGN (see Figure 13). These consistences indicate that the origin of the observed correlations in physical properties between SGN and their host galaxies can be understood in terms of dissipative SGN formation that can depend strongly on physical properties of their hosts (e.g. masses and gas mass fraction).

\subsubsection{Correlations between $m_{I}, I_{\mathrm{e}}$, and $R_{\mathrm{e}}$}

Böker et al. (2004) investigated correlations between absolute magnitudes in $I$-band $\left(m_{I}\right)$, surface brightness at effective radii $\left(I_{\mathrm{e}}\right)$ and effective radii $\left(R_{\mathrm{e}}\right)$ for $\mathrm{SGN}$ in nearby galaxies. These observations are quite useful to check how self-consistently the present SGN formation model can explain the observed properties of SGN. We assume $M / L=0.76$ in the $I$-band (corresponding to the $\mathrm{SSP}$ with $2 \mathrm{Gyr}$ and $[\mathrm{Fe} / \mathrm{H}]=0$ in Vazdekis et al. 1996) so that the simulated SGN have a range of $m_{I}$ similar to the observed one by Böker et al. (2004). Figure 15 shows that although the simulated $m_{I}-I_{\mathrm{e}}$ relation is qualitatively similar to the observed one, the simulated $I_{\mathrm{e}}$ is significantly lower than the observed one for a given $m_{I}$. The origin of this less self-consistent result can be due to the adopted larger gravitational softening length $(9-20 \mathrm{pc})$ by which formation of compact SGN can be suppressed owing to their weaker gravity.

Figure 15 also shows that the simulated $R_{\mathrm{e}}$ of SGN are significantly larger than the observed values: only two SGN observed in Böker et al. (2004) match the simulation results. The physical reason for this inconsistency with observations can be essentially the same as that for the less self-consistent result of the $m_{I}-I_{\mathrm{e}}$ relation. The simulated $m_{I}-R_{\mathrm{e}}$ shows that fainter SGN are likely to have more diffuse configurations owing to more negative feedback of SNe II (which can prevent compact stellar nuclei from forming). This tendency cannot be clearly seen in the observed $m_{I}-R_{\mathrm{e}}$ correlation. We plan to investigate whether the above inconsistency can be still seen in our future more-sophisticated numerical models with much higher numerical resolution (e.g. smaller $\epsilon_{\mathrm{g}}$ ).

\subsubsection{Number Fraction dE,Ns among $d E$ Populations}

It has long been known that more-luminous dwarf galaxies $(\mathrm{dE})$ are more likely to contain SGN (e.g. van den Bergh 1986; Freeman 1993): The number fraction of $\mathrm{dE}$,Ns among dEs is almost $100 \%$ for $M_{\mathrm{B}} \approx-18 \mathrm{mag}$ and 

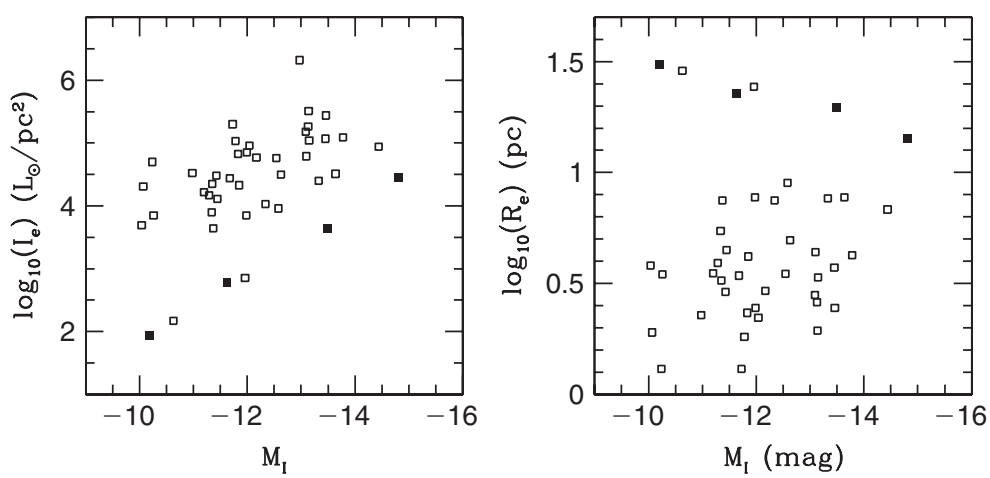

Figure 15 Correlations between $m_{\mathrm{I}}$ and $I_{\mathrm{e}}$ (left) and those between $m_{\mathrm{I}}$ and $R_{\mathrm{e}}$ (right) for observations (open squares) and simulations (filled ones). Observational data sets for 39 SGN come from Böker et al. (2004). The results of the models with different masses of stellar spheroids (M1, M11, M12, and M13) are given to show the simulated correlations between SGN with different luminosities. $I_{\mathrm{e}}$ of each simulated SGN is defined as $\log I_{\mathrm{e}}=0.4\left(4.08-m_{\mathrm{I}}\right)-\log \left(2 \pi R_{\mathrm{e}}^{2}\right)$, where $R_{\mathrm{e}}$ is given in units of parsecs (Böker et al. 2004).

about $20 \%$ for $M_{\mathrm{B}} \approx-12$ mag. Côte et al. (2006) have found that the frequency of nucleation $\left(f_{\mathrm{n}}\right)$ in early-type galaxies brighter than $M_{\mathrm{B}} \sim-15$ mag ranges from $66 \%$ to $82 \%$, though it is not observationally clear how $f_{\mathrm{n}}$ depends on $M_{\mathrm{B}}$ for faint galaxies. The present simulations have demonstrated that less-luminous galaxies can have lessremarkable SGN and some SGN can be hardly identified observationally as distinct nuclei (see Figure 11). They also have suggested that the origin of this dependence is closely associated with the fact that SGN formation is more-strongly suppressed by stronger feedback effects in less-luminous galaxies. These simulation results suggest that the origin of the observed dependence of $\mathrm{dE}, \mathrm{N}$ fraction on the host luminosities can be understood in terms of the luminosity (or mass) dependence of the effectiveness of supernovae feedback in SGN formation.

Recently Lotz et al. (2004) have revealed that some SGN of dE,Ns have colors that are significantly bluer than those of their host galaxies and can be due to recent star formation episodes. Several previous spectroscopic observations of SGN in dE,Ns also revealed possible evidence for younger stellar populations in these SGN (e.g. Caldwell \& Bothun 1987; Bothun \& Mould 1988). The present study suggests that if these apparently young SGN were formed dissipatively from gas, as our simulations have demonstrated, they should be significantly more metal-rich than their host galaxies. It is thus doubtlessly worthwhile for future spectroscopic observation to investigate the metallicity differences between SGN and their host galaxies.

Although the present results can be consistent with radial structures and kinematics of most of dE,Ns observed by Geha et al. (2002) in a self-consistent manner, they cannot be consistent with the kinematics of one of their dE,Ns (VCC 1254, see their Figure 7). The radially increasing $\sigma$ profiles are one of the characteristics of the present dissipative SGN formation and our models with central MBHs with masses consistent with the observed $M_{\mathrm{sph}}-M_{\mathrm{mbh}}$ relation (Magorrian et al. 1998) cannot simply explain the observed radially decreasing $\sigma$ profile of VCC 1254 . This inconsistency suggests that MBHs which are more massive than the $M_{\mathrm{sph}}-M_{\mathrm{mbh}}$ relation implies can play a vital role in SGN formation with radially decreasing $\sigma$ profiles.

\subsection{Origin of $\omega$ Centauri}

The observed unique physical properties of the most massive Galactic globular cluster $\omega$ Cen have long been considered to suggest that there are remarkable differences in star formation histories, chemical enrichment processes and structure formation between $\omega$ Cen and other Galactic normal globular clusters (e.g. Meylan 1987; Freeman \& Rodgers 1975; Norris, Freeman \& Mighell 1996; Norris \& Da Costa 1995; Dinescu, Girard \& van Altena 1999; Lee et al. 1999; Hilker \& Richtler 2000; Pancino et al. 2000; Smith et al. 2000; Ferraro, Bellazzini \& Pancino 2002). One of the most extensively discussed formation scenarios for $\omega$ Cen is that it is the surviving nucleus of an ancient nucleated dwarf galaxy (Zinnecker et al. 1988; Freeman 1993). Recent numerical simulations have confirmed that very massive GCs such as $\omega$ Cen in the Galaxy and G1 in M31 can be formed from tidal destruction of an ancient nucleated dwarf merging with the Galaxy and M31 (e.g. Bekki \& Freeman 2003; Bekki \& Chiba 2004; Bekki \& Norris 2006). However, these simulations do not have enough resolution to discuss the origin of the dynamical and chemical properties of the 'naked SGN' (i.e. $\omega$ Cen) of the nucleated dwarf.

This scenario the formation of $\omega$ Cen has not yet answered the following key questions related to the fundamental characteristics of $\omega$ Cen: (a) Why can $\omega$ Cen have a wide range of ages and metallicities in its stellar populations (e.g. Hilker \& Richtler 2000); (b) why is it so flattened (e.g. Meylan 1987); and (c) why is there a significant difference in kinematics between its metal-rich and metal-poor stellar populations (e.g. Norris et al. 1997). The present study has demonstrated that SGN can be formed by dissipative merging of massive clumps that have different star formation and chemical evolution histories in the central region of a galaxy. Therefore, the present study can provide the following explanations for the above three questions. 
A possible explanation for the first question is that $\omega$ Cen (i.e. SGN) was formed from dissipative merging of massive clumps with different age and metallicity distributions so that it has a very wide range of ages and metallicities in its stellar populations (see Bekki \& Freeman 2003 for a similar explanation). This explanation can also provide a clue to the origin of the age-metallicity relation of $\omega$ Cen (e.g. Hilker \& Richtler 2000) and the observed possible prolonged star formation (e.g. Villanova et al. 2007). A possible explanation for the second question is that $\omega$ Cen has a flattened shape because merging of star clusters can significantly flatten the shape of the merger remnant (e.g. Makino, Akiyama \& Sugimoto 1991; Bekki et al. 2004). This explanation means that $\omega$ Cen should be rotationally flattened (Norris et al. 1997) rather than by anisotropic velocity dispersion. An alternative explanation for the flattened shape would be that the time scale of dynamical relaxation for $\omega$ Cen is so long that $\omega$ Cen can keep its original flattened shape until recently.

An answer for the third question is that old, metal-poor stellar components of $\omega$ Cen have a significant amount of rotation, because they were already stellar components at the epoch of merging of massive clumps (i.e. at the epoch of formation of $\omega$ Cen) so that the conversion of orbital angular momentum into an intrinsic one resulted in the rotational kinematics of the old stars. Metal-rich, young and dynamically cold components in $\omega$ Cen could be formed dissipatedly from gas that was radially transfered into the central region of the merging clumps. Thus some of the observed unique physical properties of $\omega$ Cen can be understood in terms of the present dissipative SGN formation scenario.

\subsection{A Possible Evolutionary Link between SGN and UCDs}

A new type of sub-luminous and extremely compact 'dwarf galaxy' has recently been discovered in an 'allobject' spectroscopic survey centred on the Fornax cluster of galaxies (Drinkwater et al. 2000). They are observed to have intrinsic sizes of only $\sim 100 \mathrm{pc}$ and absolute $B$ band magnitudes ranging from -13 to $-11 \mathrm{mag}$ and thus are named as 'ultra-compact dwarf' (UCD) galaxies (Drinkwater et al. 2000, 2003). One of the possible scenarios is that these UCDs were previously nuclei of $\mathrm{dE}, \mathrm{Ns}$ that have lost their outer stellar envelopes by some physical processes (e.g. Drinkwater et al. 2000, 2003). The formation processes of UCDs and $\omega$ Cen are essentially the same. Recent numerical simulations have demonstrated that the strong tidal field of clusters of galaxies can remove nearly completely the outer stellar envelopes of $\mathrm{dE}, \mathrm{Ns}$ and consequently transform dE,Ns into UCDs (Bekki, Couch \& Drinkwater 2001; Bekki et al. 2003). Furthermore, recent $H S T$ observations on nuclear star clusters in spiral galaxies have revealed compact nuclei that have luminosities and sizes similar to those of UCDs (e.g. Walcher et al. 2005).

If UCDs originate from $\mathrm{dE}, \mathrm{Ns}$ and if SGN of dE,Ns were formed in such a way that the present study describes, it can be expected that UCDs should have (a) more flattened shapes, (b) rotational kinematics, and (c) younger and metal-rich stellar populations. Previous HST imaging studies of bright UCDs did not reveal any UCDs with significantly flattened shapes, though the total number of UCDs investigated was quite small (Drinkwater et al. 2003). Future and ongoing statistical studies of the ellipticity distribution of the apparent shapes of UCDs by Advanced Camera Surveys (ACS) of HST will provide a clue to the intrinsic shapes of UCDs (Drinkwater et al. 2005). Furthermore, future spectroscopic observations which enable us to estimate separately ages and metallicities of the stellar populations of UCDs will prove or disprove the predicted properties of stellar populations of UCDs (SGN). Although it is a formidable task even for a large ground-based telescope (e.g. Keck $10 \mathrm{~m}$ ) to reveal systematic rotation in integrated starlight of UCDs, such spectroscopic observations are doubtlessly worthwhile because they can support or rule out some predictions of dissipative SGN (thus UCD) formation.

\subsection{Dissipative versus Dissipationless SGN Formation}

One of the advantages of the dissipative SGN formation scenario is that it can naturally explain how massive stellar clumps, which are 'building blocks' of SGN, are formed in the central regions of gas-rich galaxies. The present study has demonstrated that massive clumps composed of gas and new stars first originate from nuclear gaseous spirals arms in a gas disk and then merge with one another to form a single massive nucleus. Although there is some evidence for the presence of spiral structures in dwarf galaxies (e.g. Jerjen, Kalnajs \& Binggeli 2000; Graham, Jerjen \& Guzmán 2003), it is not observationally clear whether nuclear gaseous spiral arms play an important role in the development of SGN in galaxies. If such nuclear nonaxisymmetric structures are important in SGN formation, as the present study demonstrates, the degree of selfgravitating of gas is a key determinant for SGN formation, because it can control the formation of non-axisymmetric structures (e.g. Binney \& Tremaine 1987).

The radial transfer of massive stellar clumps due to dynamical friction against stellar backgrounds is also a key process of SGN formation in the dissipative formation scenario. Since the time-scale of dynamical friction depends primarily on the masses of the stellar clumps, the time-scale of SGN formation can depend on the masses of the clumps that are formed in gas disks embedded by stellar spheroids. Therefore, if supernova feedback effects can strongly suppress the formation of massive clumps in gas disks, SGN formation can also be strongly suppressed. Thus the IMF and the depth of galactic gravitational potential, both of which can control the effectiveness of supernovae feedback in ISM evolution, can be key parameters of dissipative SGN formation.

\section{Conclusions}

We have numerically investigated how SGN are formed in the central region $(R<1 \mathrm{kpc})$ of gas disks embedded by 
old stellar spheroids. We summarise our principal results as follows.

1. SGN can be formed from dissipative, repeated merging of massive stellar and gaseous clumps that have typical masses of $10^{5}-10^{6} \mathrm{M}_{\odot}$ and are developed from nuclear gaseous spiral arms owing to local gravitational instability. Both gaseous self-gravity and dynamical friction are important physical processes in dissipative SGN formation.

2. SGN just after their formation are not located in the very center of their host spheroids and dynamical friction between SGN and stellar backgrounds can transfer them into the very mass center of their hosts. Typically only $\sim 5 \%$ of the speroidal masses can be converted into SGN from gas clouds.

3. SGN have very flattened shapes, rotational kinematics and radially increasing $\sigma$ (velocity dispersion) profiles (lower $\sigma$ in the inner region). These structural and kinematic characteristics do not depend on model parameters such as masses of spheroids $\left(M_{\mathrm{sph}}\right)$ and initial gas mass fraction $\left(f_{\mathrm{g}}\right)$.

4. Stellar populations of SGN can show a wide range of ages and metallicities, because SGN are formed from massive clumps with different star-formation and chemical-evolution histories. The mean metallicity of SGN can be significantly higher than that of the original gas disks (thus than those of their host spheroids).

5. The central massive black holes $(\mathrm{MBH})$ with $M_{\mathrm{mbh}} \approx 0.01 M_{\mathrm{sph}}$ do not influence the formation processes of SGN. IMF of star-forming clouds can significantly influence SGN formation in the sense that SGN formation is strongly suppressed for the top-heavy IMFs.

6. More massive, higher density SGN can be formed in spheroids with higher surface brightness. Furthermore there can be a threshold value $(\sim 0.2)$ of $f_{\mathrm{g}}$ below which massive SGN are less likely to be formed in the central gas disks of spheroids. If higher-surface-brightness galaxies were formed at higher redshifts, the above result implies that $\mathrm{dE}$,Ns could be earlier virialized objects than dEs (without SGN).

7. More-massive spheroids can have more-massive, more metal-rich and higher-density SGN, because star formation and chemical enrichment proceeds more efficiently in more-massive spheroids owing to the lessdramatic suppression of star formation by supernova feedback effects in more-massive spheroids. Lessmassive spheroids are likely to have younger SGN owing to the longer time-scales of SGN formation.

Based on these results, we have discussed how selfconsistently the dissipative SGN formation scenario can explain key observations of SGN. We also have demonstrated that the formation of nuclear gaseous spiral arms, the self-gravity of gas and the dynamical friction between massive clumps and stellar backgrounds are key determinants in dissipative SGN formation. It has also been remarked that theoretical/numerical studies such as those done in the present study can also provide some clues to the origin of massive globular clusters (e.g. $\omega$ Centauri and UCDs).

\section{Acknowledgments}

We are grateful to the referee for valuable comments, which contribute to improve the present paper. K.B. acknowledges Yasuhiro Shioya for his useful discussions on chemodynamical evolution of low-mass galaxies. K.B. acknowledges the financial support of the Australian Research Council throughout the course of this work. The numerical simulations reported here were carried out on GRAPE systems kindly made available by the Astronomical Data Analysis Center (ADAC) at the National Astronomical Observatory of Japan (NAOJ).

\section{References}

Andredakis, Y. C. \& Sanders, R. H., 1994, MNRAS, 267, 283

Balcells, M., Graham, A. W., Domínguez-Palmero, L. \& Peletier, R. F., 2003, ApJ, 582, 79

Bekki, K. \& Shioya, Y., 1998, ApJ, 497, 108

Bekki, K. \& Shioya, Y., 1999, ApJ, 513, 108

Bekki, K. \& Freeman, K. C., 2003, MNRAS, 346, L11

Bekki, K., Couch, W. J. \& Drinkwater, M. J., 2001, ApJ, 552, L105

Bekki, K., Couch, W. J., Drinkwater, M. J. \& Shioya, Y., 2003, MNRAS, 344, 399

Bekki, K., Couch, W. J., Drinkwater, M. J. \& Shioya, Y. 2004, ApJ, 610, L13

Bekki, K. \& Chiba, M., 2004, A\&A, 417, 437

Bekki, K. \& Norris, J. E., 2006, ApJL, 637, 109

Bekki, K., Couch, W. J. \& Shioya, Y., 2006, ApJL, 642, 133

Bekki, K., Shioya, Y. \& Whiting, M., 2006, MNRAS, 371, 805

Binggeli, B. \& Cameron, L. M., 1991, A\&A, 252, 27

Binney, J. \& Tremaine, S., 1987, Galactic Dynamics (Princeton, NJ: Princeton University Press)

Binney, J. \& Merrifield, M., 1998, Galactic Astronomy (Princeton, NJ: Princeton University Press)

Böker, T., Laine, S., van der Marel, R. P., Sarzi, M., Rix, H.-W., Ho, L. C. \& Shields, J. C., 2002, AJ, 123, 1389

Böker, T., Sarzi, M., McLaughlin, D. E., van der Marel, R. P., Rix, H.-W., Ho, L. C. \& Shields, J. C., 2004, AJ, 127, 105

Bothun, G. D. \& Mould, J. R., 1988, ApJ, 324, 123

Caldwell, N. \& Bothun, G. D., 1987, 94, 1126

Carollo, C. M., Stiavelli, M. \& Mack, J., 1998, AJ, 116, 68

Combes, F. \& Gerin, M., 1985, A\&A, 150, 327

Côte, P., Marzke, R. O., West, M. J. \& Minniti, D., 2000, ApJ, 533, 869

Côte, P. et al., 2006, ApJS, 165, 57

Davies, R. L., Burstein, D., Dressler, A., Faber, S. M., Lynden-Bell, D., Terlevich, R. J. \& Wegner, G., 1987, ApJS, 64, 581

Dekel, A. \& Silk, J., 1986, ApJ, 303, 39

De Propris, R., Philipps, S., Drinkwater, M. J., Gregg, M. D., Jones, J. B., Evstigneeva, E. \& Bekki, K., 2005, ApJL, 623, 105

Dinescu, D. I., Girard, T. M. \& van Altena, W. F., 1999, AJ, 117, 1792

Djorgovski, S. G., Gal, R. R., McCarthy, J. K., Cohen, J. G., de Carvalho, R. R., Meylan, G., Bendinelli, O. \& Parmeggiani, G., 1997 ApJ, 474, L19

Drinkwater, M. J., Jones, J. B., Gregg, M. D. \& Philipps, S. 2000, PASA, 17, 227

Drinkwater, M. J., Gregg, M. D., Hilker, M., Bekki, K., Couch, W. J., Ferguson, J. B., Jones, J. B. \& Philipps, S., 2003, Nature, 423, 519

Drinkwater, M. J. et al., 2005, submitted to AJ

Elmegreen, B. G., 2002, Ap\&SS, 284, 819

Fellhauer, M. \& Kroupa, P., 2002, Ap\&SS, 281, 355 
Ferguson, H. C. \& Bingelli, B., 1994, A\&ARv, 6, 67

Ferraro, F. R., Bellazzini, M. \& Pancino, E., 2002, ApJL, 573, 95

Freeman, K. C. \& Rodgers, A. W., 1975, ApJ, 201, 71

Freeman, K. C. 1993, in ASPC 48, The Globular Clusters-Galaxy Connection, Eds. Smith, G. H. \& Brodie, J. P. (San Francisco: Astronomical Society of the Pacific), 48, 608

Geha, M., Guhathakurta, P. \& van der Marel, R. P., 2002, AJ, 124, 3073

Graham, A. W., Jerjen, H. \& Guzmán, R., 2003, AJ, 126, 1787

Graham, A. W. \& Guzmán, R., 2003, AJ, 125, 2936

Gregg, M. D. et al. 2007, submitted to AJ

Hausman, M. A. \& Roberts, W. W., Jr., 1984, ApJ, 282, 106

Hilker, M. \& Richtler, T., 2000, A\&A, 362, 895

Ichikawa, S. I., Wakamatsu, K. I. \& Okamura, S., 1986, ApJS, 60, 475

Jerjen, H., Kalnajs, A. \& Binggeli, B., 2000, A\&A, 358, 845

Jones, J. B. et al., 2006, AJ, 131, 312

Kauffmann, G. et al., 2003, MNRAS, 341, 54

Kennicutt, R. C., Jr., 1998, ARA\&A, 36, 189

Lambas, D. G., Maddox, S. J. \& Loveday, J. 1992, MNRAS, 258, 404

Larson, R. B., 1981, MNRAS, 194, 809

Larson, R. B., 1998, MNRAS, 301, 569

Lee, Y.-W., Joo, J.-M., Sohn, Y.-J., Rey, S.-C., Lee, H.-C. \& Walker, A. R., 1999, Natur, 402, 55

Lotz, J. M., Miller, B. W. \& Ferguson, H. C., 2004, ApJ, 613, 262

Magorrian, J. T. et al., 1998, AJ, 115, 2285

Makino, J., Akiyama, K. \& Sugimoto, D., 1991, Ap\&SS, 185, 63

Mateo, M., 1998, ARAA, 36, 435

Matthews, L. D. et al., 1999, AJ, 118, 208

Meylan, G., 1987, A\&A, 184, 144

Mieske, S., Hilker, M. \& Infante, L., 2004, A\&A, 418, 445

Milosavljević, M., 2004, ApJ, 605, L13

Mould, J. R., 1984, PASP, 96, 773
Noguchi, M. 1991, IAUS, 146, 343

Norris, J. E., Freeman, K. C. \& Mighell, K. J., 1996, ApJ, 462, 241

Norris, J. E., Freeman, K. C., Mayor, M. \& Seitzer, P., 1997, ApJ, 487, L187

Norris, J. E. \& Da Costa, G. S., 1995, ApJ, 447, 680

Oh, K. S. \& Lin, D. N. C., 2000, ApJ, 543, 620

Pancino, E., Ferraro, F. R., Bellazzini, M., Piotto, G. \& Zoccali, M., 2000, ApJL, 534, 83

Philips, S., Drinkwater, M. J., Gregg, M. D. \& Jones, J. B., 2001, ApJ, 560, 201

Phillips, A. C., Illingworth, G. D., MacKenty, J. W. \& Franx, M. 1996, AJ, 111, 1566

Seth, A. C., Dalcanton, J. J., Hodge, P. W. \& Debattista, V. P., 2006, AJ, 132, 2539

Schmidt, M., 1959, ApJ, 129, 243

Smith, V. V., Suntzeff, N. B., Cunha, K., Gallino, R., Busso, M., Lambert, D. L. \& Straniero, O., 2000, AJ, 119, 1239

Stiavelli, M., Miller, B. W., Ferguson, H. C., Mack, J., Whitmore, B. C. \& Lotz, J. M., 2001, AJ, 121, 1385

Sugimoto, D., Chikada, Y., Makino, J., Ito, T., Ebisuzaki, T. \& Umemura, M., 1990, Natur, 345, 33

Tacconi, L. J. \& Young, J. S., 1986, ApJ, 308, 600

Thornton, K., Gaudlitz, M., Janka, H.-Th. \& Steinmetz, M., 1998, ApJ, 500, 95

Tremaine, S. D., Ostriker, J. P. \& Spitzer, L., Jr., 1975, ApJ, 196, 407 van den Bergh, S., AJ, 1986, 91, 271

Vazdekis, A., Casuso, E., Peletier, R. F. \& Beckman, J. E., 1996, ApJS, 106, 307

Villanova, S. et al., 2007, ApJ, 663, 296

Walcher, C. J. et al., 2005, ApJ, 618, 237

Zinnecker, H., Keable, C. J., Dunlop, J. S., Cannon, R. D. \& Griffiths, W. K., 1988, in Globular Cluster Systems in Galaxies, Eds. Grindlay, J. E. \& Davis Philip, A. G. (Dordrecht, Kluwer), 603 